\title{
The lymphoid-associated interleukin 7 receptor (IL-7R) regulates tissue resident macrophage development
}

Gabriel A. Leung ${ }^{1 \dagger}$ Taylor Cool, ${ }^{2,3, \dagger}$ Clint H. Valencia ${ }^{4}$, Atesh Worthington ${ }^{2}$, Anna E. Beaudin $^{4 \dagger *}$, E. Camilla Forsberg ${ }^{2 \dagger * *}$

${ }^{1}$ Quantitative and Systems Biology Program, University of California-Merced, Merced, CA, USA

${ }^{2}$ Institute for the Biology of Stem Cells, University of California-Santa Cruz, Santa Cruz, CA, USA

${ }^{3}$ Department of Biological Sciences, San Jose State University, San Jose, CA, USA

${ }^{4}$ Molecular and Cell Biology Department, School of Natural Sciences, University of California-

Merced, Merced, CA, USA

${ }^{\dagger}$ These authors contributed equally to this work.

$\dagger$ These authors contributed equally to this work.

* Correspondence: cforsber@ucsc.edu, abeaudin@ucmerced.edu 


\begin{abstract}
The discovery of a fetal origin for tissue-resident macrophages (trMacs) has inspired an intense search for the mechanisms underlying their development. Here, we performed in vivo lineage tracing of cells with an expression history of IL-7R $\alpha$, a marker exclusively associated with the lymphoid lineage in adult hematopoiesis. Surprisingly, we found that IL7R-Cre labeled fetalderived, adult trMacs. Labeling was almost complete in some tissues and partial in other organs. The putative progenitors of trMacs, yolk sac (YS) erythromyeloid progenitors (EMPs), did not express IL-7R, and YS hematopoiesis was unperturbed in IL-7R-deficient mice. In contrast, tracking of IL-7R $\alpha$ message levels, surface protein expression, and IL7R-Cre-mediated labeling across fetal development revealed dynamic regulation of IL-7R $\alpha$ mRNA expression and rapid upregulation of IL-7R $\alpha$ surface protein upon transition from monocyte to macrophage within fetal tissues. Fetal liver monocyte differentiation in vitro produced IL-7R+ macrophages, supporting a direct progenitor-progeny relationship. Additionally, blockade of IL-7R function during late gestation specifically impaired the establishment of fetal-derived tissue macrophages in vivo. These data provide evidence for a distinct function of IL-7R $\alpha$ in fetal myelopoiesis and identify IL-7R as a novel regulator of tissue-resident macrophage development.
\end{abstract}

\title{
Introduction
}

Hematopoietic stem cells (HSCs) are responsible for sustaining blood and immune cell production across the lifespan of the animal, under steady-state conditions, during infection, and following transplantation. However, recent findings have revealed that adult HSCs have a limited capability to generate or regenerate many tissue-resident immune cells, including subsets of tissue-resident macrophages (trMacs) such as microglia, epidermal Langerhans cells, liver Kupffer cells, and alveolar macrophages (Beaudin and Forsberg, 2016; Cool and Forsberg, 2019; Ginhoux et al., 2010; Gomez Perdiguero et al., 2015; Guilliams et al., 2013; Hashimoto et al., 2013; Hoeffel et al., 2012; Sawai et al., 2016; Yona et al., 2013). TrMacs are specialized macrophages that reside within tissues and have specific functions in tissue and immune homeostasis (Epelman et al., 2014; Ginhoux et al., 2010; Gomez Perdiguero et al., 2015; Guilliams et al., 2013; Hoeffel et al., 2012). Unlike classical adult monocyte-derived macrophages that circulate and have high turnover rates, trMacs are maintained via local 
proliferation within specific tissues, largely independent of contribution from adult hematopoiesis (Ajami et al., 2007; Hashimoto et al., 2013; Hulsmans et al., 2017; Merad et al., 2002). Recent fate mapping studies have provided direct evidence for a fetal origin of specific trMacs (Epelman et al., 2014; Gomez Perdiguero et al., 2015; Hoeffel et al., 2015; Schulz et al., 2012; Yona et al., 2013). However, the cellular and molecular mechanisms driving trMac establishment and expansion within fetal tissues are poorly understood.

Accumulating evidence points towards extraembryonic yolk sac (YS)-derived erythromyeloid progenitors (EMPs) as the initial cell-of-origin for macrophages that seed developing tissues, and the primary source of brain microglia (Ginhoux et al., 2010; Gomez Perdiguero et al., 2015; Kierdorf et al., 2013). Microglia and other trMacs can differentiate directly from EMPs through a myb-independent pathway or through a myb-dependent pathway, likely through an EMP-derived intermediate (Hoeffel et al., 2015; Schulz et al., 2012). In certain fetal-derived trMac populations, including lung alveolar macrophages, epidermal Langherhans cells, and liver Kupffer cells, macrophages derived from fetal liver (FL)-derived precursors may replace or replenish initially seeded YS-derived macrophages (Guilliams et al., 2013; Hoeffel et al., 2015; Yona et al., 2013). However, both the precise cell of origin for FL precursors and their specific contribution to adult trMac compartments remain controversial, due in part to incomplete progenitor labeling and the difficulties in accurately tracking progeny in situ. Despite intense investigation of the mechanisms regulating macrophage differentiation from YS progenitors, including gene expression programs (Mass et al., 2016) and mechanisms of tissue seeding (Stremmel et al., 2018), comparatively less is known about the developmental mechanisms that regulate the establishment of tissue macrophages from later waves of hematopoietic cell production.

Here, our investigation of hematopoietic development using the IL7R-Cre lineage tracing model (Schlenner et al., 2010) revealed unexpected and robust labeling of adult trMacs in multiple tissues. Examination of fetal myeloid development revealed a transient wave of IL-7R $\alpha$ expression on developing fetal macrophages that was dynamically regulated as macrophages differentiated within developing resident tissues. Both germline deletion and specific blockade of the IL-7R during the developmental window in which fetal tissue macrophages express IL-7R 
confirmed the functional requirement for IL7R signaling during fetal trMac development. In contrast, IL-7R $\alpha$ was not expressed by YS EMPs and yolk sac hematopoiesis was unperturbed in IL 7R $\mathrm{R}^{-/-}$embryos. Together, these experiments reveal IL-7R as a novel regulator of fetal macrophage development during late gestation.

\section{Results}

\section{IL7R-Cre specifically labels adult tissue-resident macrophages}

The lymphoid-associated genes Flk2, IL-7R $\alpha$ and Rag1 label cells with increasingly restricted lymphoid potential in adult hematopoiesis (Adolfsson et al., 2005; Forsberg et al., 2006; Igarashi et al., 2002; Kondo et al., 1997). In contrast, all three mark oligopotent hematopoietic progenitors with both myeloid and lymphoid potential during fetal development (Beaudin et al., 2016; Boiers et al., 2013). While we and others have used the Flk2-Cre and Rag1-Cre models to track the contribution of fetal progenitors to tissue resident macrophage (trMac) populations (Boiers et al., 2013; Epelman et al., 2014; Gomez Perdiguero et al., 2015; Hashimoto et al., 2013; Hoeffel et al., 2015), the contribution of IL7R-marked progenitors to the same populations has not been previously examined. To decipher the contribution of specific transient hematopoietic progenitors to adult trMacs, we compared trMac labeling across three lineage tracing models: Flk2-Cre, IL7R-Cre, and Rag1-Cre. We crossed mice expressing Rag1-Cre (Welner et al., 2009) or IL7R-Cre (Schlenner et al., 2010) to mTmG mice expressing a dual color fluorescent reporter (Muzumdar et al., 2007), thereby creating "Rag1Switch" and IL7RSwitch" models (Fig.1A) analogous to the previously described FlkSwitch mouse (Beaudin et al., 2016; Boyer et al., 2012; Boyer et al., 2011). In all models, all cells express Tomato (Tom) until Cremediated recombination results in the irreversible switch to GFP expression by that cell and all of its progeny (Fig.1B).

We compared reporter expression in fetal-derived trMacs of all three models to reporter expression in circulating peripheral blood (PB) monocytes $\left(\mathrm{CD} 11 \mathrm{~b}^{\text {hi }} \mathrm{Gr} 1^{\text {lo }}\right)$, the precursors of adult HSC-derived macrophages. As expected, Cre-driven GFP labeling of monocytes in IL 7R $\alpha$ Switch and Rag1Switch mice was less than 5\% (Fig.1C) and paralleled the nominal labeling observed in adult HSCs and myeloid progenitors (Fig. S1A,B), as previously reported 
(Schlenner et al., 2010; Welner et al., 2009). Adult FlkSwitch mice exhibited high GFP labeling in PB monocytes (Figure 1C), as we previously reported (Boyer et al., 2011). Intriguingly, examination of Cre-driven reporter switching in adult trMacs revealed distinct GFP labeling patterns across all three lineage tracing models and across tissues. Within Langerhans cells (LCs) of the epidermis, around 20\% of cells were labeled by GFP in the FlkSwitch model (Fig. 1D), consistent with previous reports (Gomez Perdiguero et al., 2015; Hoeffel et al., 2015). Low GFP labeling was observed in LCs of Rag1Switch mice (Fig. 1D), as described previously for skin and other tissue macrophages at E14.5 (Boiers et al., 2013). In sharp contrast, up to 96\% of LCs expressed GFP in IL7RSwitch mice (Fig.1D). A similar pattern was observed for microglia; consistent with a pre-HSC origin, virtually no reporter switching was observed in adult microglia of FlkSwitch mice (Fig.1E). Minimal microglia labeling was also observed in Rag1Switch mice. Remarkably, however, GFP-labeling of microglia was over $85 \%$ in IL7RSwitch mice (Fig.1E).

Examination of reporter expression in trMacs of the lung (alveolar macrophages, AMs) and the liver (Kupffer cells, KCs) yielded another labeling pattern across lineage tracing models, as compared to the LC and microglia (Fig.1F,G). In FlkSwitch and Rag1Switch mice, GFP labeling in AMs and KCs was low and roughly comparable to LCs ( 8-19\% GFP+ cells; Fig. 1D,F,G). Surprisingly, in IL7RSwitch mice, GFP labeling in AM and KC populations was substantially higher, $40 \%$, as compared to both FlkSwitch and Rag1Switch models (Fig.1F,G). Although labeling in these two populations was still considerably less than that of LC or microglia in the same mice ( $~ 85-96 \%)$, it was substantially higher than labeling of adult BM-derived myeloid populations $\left(\mathrm{F} 4 / 80^{\mathrm{lo}} \mathrm{CD} 11 \mathrm{~b}+\right)$ within the same tissues $(<5 \%)$ (Fig. S1C-E). Comparison of Cremediated labeling between circulating BM-derived monocytes (4\%) and trMacs (40-90\%) in adult IL7RSwitch mice also revealed stark differences in labeling, supporting previous reports that adult BM-derived monocytes did not substantially contribute (at steady-state) to the trMac populations investigated, including microglia, Langerhans cells, Kupffer cells, and alveolar macrophages (Gomez Perdiguero et al., 2015; Hashimoto et al., 2013; Hoeffel et al., 2012; Sheng et al., 2015). Despite substantial IL7R-Cre-mediated labeling, IL-7R $\alpha$ surface expression was undetectable in any adult trMacs surveyed (Fig. 1H). IL-7R $\alpha$ message was also virtually undetectable in trMacs as compared to BM Pro-B-cells (Figure 1I). The robust labeling of adult 
trMacs in the IL7R $\alpha$ Switch model therefore suggested IL-7R $\alpha$ expression during an earlier window of macrophage development.

\section{YS hematopoiesis does not depend on IL7R}

Yolk sac (YS) erythromyeloid progenitors (EMPs) have been proposed as a cell of origin for fetal trMac development, either through direct differentiation or through the myb-dependent generation of intermediate progenitors that seed the fetal liver (Gomez Perdiguero et al., 2015; Hoeffel et al., 2015; Hoeffel et al., 2012; Mass et al., 2016; Schulz et al., 2012). To determine whether IL7R-Cre labeling of adult trMac populations initiated in YS EMPs during embryonic development, we examined IL7R-Cre driven GFP labeling, IL-7R $\alpha$ surface expression and IL7R $\alpha$ message in YS EMPs and YS macrophages. YS EMPs (Ter119-cKit+CD41+) at E9.5 were minimally labeled by IL7R-Cre, consistent with a complete absence of IL-7R $\alpha$ surface expression (Fig 2A). IL-7R $\alpha$ message was detectable at very high $\mathrm{Ct}$ values (Fig $2 \mathrm{C}, \mathrm{C}^{\prime}$ ), and consequently we observed slightly more labeling in YS EMPs by E10.5 (Fig. 2A). However, increased reporter expression was not accompanied by surface expression (Fig. 2A'). Labeling of YS EMPs was comparable to background labeling observed in adult hematopoiesis (Schlenner et al., 2010). Few YS myeloid cells, defined by either CD11b or F4/80 expression within the whole CD45+ fraction, exhibited IL7R-Cre driven reporter expression (Fig. 2B) and they did not express surface IL-7R $\alpha$ (Fig. 2B'). To determine whether IL-7R expression regulated YS hematopoiesis, we examined the frequency of YS EMPs and YS macrophages in IL7R-/embryos. Neither YS EMPs nor YS macrophage frequency was significantly different between WT and IL7R-/- mice (Fig. 2D,E). Together, these data indicate that IL-7R is not required for the generation of YS progenitors or YS macrophages, and suggest that labeling of F4/80 ${ }^{\text {hi }}$ trMacs by IL 7R-Cre occurs later in trMac ontogeny.

\section{IL 7R $\alpha$-Cre labels non-HSC progenitors in the fetal liver}

As early YS progenitors and macrophages were minimally labeled by IL7R-Cre, we investigated labeling in putative progenitors slightly later in development. Labeling remained comparably low in both YS and AGM cKit+CD41+ EMPs at E11.5, and IL-7R surface expression was still undetectable in the EMPs within the FL (Fig. 2A, Fig. S2A). Higher IL7R-Cre driven labeling was observed in FL Lin-cKit+Sca1+ (KLS) progenitors by E12.5, but surface expression was 
still low (Fig. 2F, H). Labeling subsequently declined in E14.5 progenitors (Fig. 2G, H; p < 0.001), and all labeling in E14.5 progenitors was confined to the CD150- multipotent progenitor compartment (Fig. S2B). Consistent with labeling of upstream multipotent cells in the FL, comparable IL7R-Cre labeling was observed in all downstream progenitor and mature compartments (Fig S2C-G), with the exception of significant labeling in committed lymphoid progenitors (Fig. S2D) and mature lymphoid cells (Fig S2E). Labeled progenitors at E14.5 did not possess long-term multilineage reconstitution, confirming that they were not definitive HSCs (Fig. S2H). These data suggest that IL-7R reporter expression in late (E10.5) YS and AGM partially labels a transient progenitor that contributes to FL hematopoiesis but is not a definitive HSC.

\section{IL7R-Cre labeling of myeloid cells is tissue- and developmental stage-specific}

Given that IL7R-Cre labeling in adult trMacs (Fig. 1) was considerably higher as compared to any progenitor we profiled across fetal development (Fig. 2H; Fig. S2), we reasoned that progenitor labeling alone could not entirely explain labeling observed in those compartments. To resolve this discrepancy, we next evaluated IL7R-Cre-driven labeling and IL-7R $\alpha$ expression in myeloid-restricted precursors within peripheral tissues during FL stage development. We initiated our investigation at E12.5, the stage at which significant progenitor labeling by IL7RCre was initially observed (Fig. 2F,H). At this stage of development, macrophages derived from the extra-embryonic $\mathrm{YS}\left(\mathrm{F} 4 / 80^{\mathrm{hi}} \mathrm{CD} 11 \mathrm{~b}^{\mathrm{lo}}\right)$ are already present in the tissues, but may be replaced by incoming FL-derived macrophage precursors or monocytes $\left(\mathrm{F} 4 / 80^{\mathrm{lo}} \mathrm{CD} 11 \mathrm{~b}{ }^{\mathrm{hi}}\right.$, Fig. $\left.3 \mathrm{~A}\right)$. $\mathrm{F} 4 / 80^{1 \mathrm{lo}} \mathrm{CD} 11 \mathrm{~b}^{\mathrm{hi}}$ macrophage precursors in fetal peripheral tissues are heterogeneous or low for Ly6c expression and express the fetal macrophage marker CD64 (Fig. S3A; (Hoeffel et al., 2015), suggesting their propensity to differentiate into macrophages.

Analysis of IL7R-Cre driven reporter expression revealed two very clear patterns: first, GFP labeling at E12.5 was significantly higher in all F4/80 ${ }^{\mathrm{lo}}$ monocytes as compared to F4/80 ${ }^{\mathrm{hi}}$ macrophages across all tissues (Fig/ 3B, left plot/E12.5 data). Second, cross-tissue comparison revealed higher GFP labeling in F4/80 ${ }^{\mathrm{lo}}$ monocytes in brain, lung, and epidermis as compared to liver (Figure 3B, left plot/E12.5 data). Across all F4/80 hi macrophages, GFP labeling was highest in the skin $\left(25 \% ; \mathrm{P}<0.05\right.$ compared to liver $\mathrm{F} 4 / 80^{\text {hi }}$ macrophages $)$. A very comparable pattern 
emerged at E14.5; however, between E12.5 and E14.5, GFP labeling increased slightly across all F4/80 ${ }^{\text {hi }}$ macrophage populations, but only significantly for the skin (Fig. 3C, Epidermis panel).

From late gestation into early neonatal development, the patterns of IL7R-Cre mediated GFP labeling among F4/80 ${ }^{\mathrm{hi}}$ macrophages and $\mathrm{F} 4 / 80^{\mathrm{lo}}$ monocytes were strikingly different, both between cell types and between tissues (Fig. 3B, C). Beyond E17.5, GFP labeling in liver and lung was higher in F4/80 $0^{\text {hi }}$ macrophages as compared to F4/80 ${ }^{\text {lo }}$ monocytes. This "switch" reflected a significant increase in GFP labeling of F4/80 ${ }^{\text {hi }}$ macrophages between E14.5 and P0 and a concomitant decrease in F4/80 ${ }^{\mathrm{lo}}$ monocyte labeling (Fig. 3C), coincident with the HSCdependent contribution to fetal monocytes later in gestation (Hoeffel et al., 2015; Yona et al., 2013). GFP labeling in lung and liver macrophages reached adult levels by P0 (Fig 3B, Figure 1F,G; P > 0.5). These data suggested that IL7R-Cre labeling was either occurring cellautonomously in F4/80 ${ }^{\mathrm{hi}}$ macrophages or that IL7R-labeled fetal monocytes were gradually replacing tissue macrophage populations in the lung and liver during fetal hematopoiesis.

In contrast to lung and liver, progression of GFP labeling within the skin and brain across ontogeny relayed a different pattern. Monocytes were barely detectable in the epidermis and brain after E14.5 (Fig. 3A, bottom right panels), as previously described (Hoeffel et al., 2015). GFP labeling of skin macrophages increased robustly from E17.5 to P0 (Fig. 3C, skin), and continued to increase postnatally (Fig. 3B,C). Similarly, microglia labeling increased steadily across development, but had still not reached adult levels by postnatal day 14 (P14) (Fig. 3B,C). The disparate GFP labeling that we observed across different tissues suggested a differential involvement of IL-7R in the development of different trMac populations across ontogeny.

\section{IL-7R $\alpha$ expression is dynamically regulated during fetal tissue macrophage development}

To gain insight into the observed pattern of IL7R-Cre mediated GFP labeling within F4/80 ${ }^{\text {lo }}$ monocyte and F4/80 ${ }^{\text {hi }}$ macrophage populations across development, we probed IL-7R $\alpha$ message levels beginning at E14.5 (Fig. 4A). Across tissues, F4/80 lo monocytes expressed low but consistently detectable levels of IL-7R $\alpha$ message relative to fetal liver monocyte dendritic progenitors (MDPs) that are known to robustly express IL-7R $\alpha$ message (Hoeffel et al., 2015)Fig. 4A). Circulating peripheral blood Ly6c+ monocytes also expressed detectable IL-7R $\alpha$ 
message and were labeled by IL7R-Cre to a similar degree as compared to liver F4/80 ${ }^{\text {lo }}$ monocytes (Fig S3C,D). Increased IL-7R $\alpha$ mRNA in peripheral tissue F4/80 $0^{\mathrm{lo}}$ monocytes corresponded with higher Cre-mediated GFP expression (Fig. 4A), as GFP labeling increased in monocytes once they had exited the liver at E14.5 (Fig. 3B). Lung and skin monocytes with the highest GFP labeling also expressed the highest levels of IL7R $\alpha$ mRNA. In comparison, and consistent with significantly lower Cre-mediated GFP labeling, IL-7R $\alpha$ message was virtually undetectable in F4/80 macrophages (Figure 4A) during the prenatal period. Postnatally, at P14, IL-7R $\alpha$ message levels continued to be negligible in macrophages of the lung and liver, but microglia and LCs expressed IL-7R $\alpha$ message (Fig. 4B), driving continued postnatal Cremediated recombination in those tissues. These data confirmed the fidelity of the IL7R-Cre model and revealed IL7R expression by fetal myeloid cells during development.

To ascertain the relationship between IL7R-Cre mediated GFP labeling and IL-7R $\alpha$ surface expression in developing macrophages during fetal development, we profiled surface expression from E12.5-P14. As GFP labeling and IL7Ra mRNA expression was higher in F4/80 ${ }^{\text {lo }}$ monocytes as compared to $\mathrm{F} 4 / 80^{\text {hi }}$ macrophages during fetal development, we expected to observe monocytes displaying surface IL7R. Unexpectedly, F4/80 ${ }^{\text {lo }}$ monocytes never displayed detectable IL-7R $\alpha$ surface protein at any timepoints examined (Fig. 4C). Surprisingly, robust IL$7 \mathrm{R} \alpha$ surface protein was instead observed on prenatal F4/80 $\mathrm{hi}$ macrophages in peripheral tissues (lung, brain, and skin), whereas minimal surface expression was observed on F4/80 hi macrophages in the liver (Fig. 4D). IL-7R $\alpha$ surface protein on peripheral F4/80 hi trMacs was observed beginning at E12.5, and peaked at E17.5 (Figure 4B, middle panel), despite significantly lower GFP labeling as compared to F4/80 ${ }^{\mathrm{lo}}$ monocytes (Fig. 3B,C). The vast majority ( $>90 \%$ ) of IL-7R $\alpha$-expressing macrophages in the brain, lung, and skin also coexpressed the common $\gamma$ chain (Fig. S4). By P0, macrophages in the liver and lung ceased to express IL-7R $\alpha$, concomitant with a plateau in GFP labeling by birth (Fig.3C). In contrast, some proportion of macrophages in the brain and epidermis continued to express IL-7R $\alpha$ protein at the surface postnatally, albeit at lower levels, and IL-7R $\alpha$ surface expression was detectable on epidermal macrophages as late as P14. Continued IL-7R $\alpha$ expression in these particular tissues was consistent with continued postnatal labeling by IL7R-Cre (Fig.3C) and IL-7R $\alpha$ mRNA 
expression (Fig. 4B). These data therefore revealed tightly regulated and highly coordinated expression of IL-7R $\alpha$ in developing macrophages across different tissues.

\section{IL-7R $\alpha$-expressing monocytes give rise to IL-7R+ macrophages ex vivo}

We hypothesized that $\mathrm{F} 4 / 80^{\mathrm{lo}} \mathrm{CD} 11 \mathrm{~b}^{\text {hi }}$ monocytes upregulate IL-7R $\alpha$ message as they exit the liver and migrate to and enter fetal tissues, where they differentiate into F4/80 ${ }^{\text {hi }}$ macrophages. IL-7R $\alpha$ surface expression is then rapidly switched on as $\mathrm{F} 4 / 80^{\mathrm{lo}}$ monocytes differentiate into $\mathrm{F} 4 / 80^{\mathrm{hi}}$ macrophages. To test our hypothesis, we isolated $\mathrm{F} 4 / 80^{\mathrm{lo}}$ monocytes from the fetal liver at E14.5 and differentiated them into F4/80 ${ }^{\text {hi }}$ macrophages ex vivo in the presence of M-CSF. $\mathrm{F} 4 / 80^{\text {lo }} \mathrm{CD} 11 \mathrm{~b}^{\text {hi }}$ cells were sorted from FL based on Ly6C expression (Fig. 5A) and cultured with 20ng M-CSF/mL for 5 days. FL monocytes expressed detectable IL-7R $\alpha$ message (Fig. 4A), but IL-7R surface expression was not observed (Fig 5C, $C^{\prime}$ ). M-CSF induced the differentiation of Ly6c ${ }^{\text {hi }} \mathrm{CD} 11 \mathrm{~b}^{\text {hi }}$ cells to $\mathrm{F} 4 / 80^{\text {hi }} \mathrm{CD} 11 \mathrm{~b}+$ macrophages that expressed CD64 after only 1 day in culture, and macrophage differentiation plateaued at 3 days (Fig 5C,E, E'). Remarkably, differentiated F4/80 ${ }^{\text {hi }}$ cells also upregulated IL-7R $\alpha$ expression on the cell surface (Fig. 5C, $\mathrm{C}^{\prime}$ ). IL-7R $\alpha$ was co-expressed with the common gamma chain, indicating expression of a functional IL-7R receptor (Fig. 5D, D'). In contrast to Ly $6 c^{\text {hi }}$ cells, Ly $6 c^{10} C D 11 b^{\text {hi }}$ FL monocytes displayed limited differentiation into F4/80 ${ }^{\mathrm{hi}}$ macrophages, even after 5 days in culture with M-CSF (Fig. $\mathrm{S} 3 \mathrm{~F}$ ), and both MDP and Ly $6 \mathrm{c}^{\mathrm{lo}}$ monocytes displayed significantly higher Cre-mediated labeling as compared to Lyc $6^{\text {hi }}$ monocytes (Fig. S3C), suggesting distinct developmental pathways. These data reveal that Ly $6 c^{\text {hi }} \mathrm{CD} 11 \mathrm{~b}^{\text {hi }}$ cells in the fetal liver that express IL-7R $\alpha$ message (Fig. 4A; Fig S3D) but not surface IL-7R surface protein (Fig. S3E) have the capacity to differentiate into $\mathrm{F} 4 / 80^{\mathrm{hi}}$ macrophages, and that this differentiation is accompanied by upregulation of surface IL-7R expression.

\section{IL-7 $\mathrm{R} \alpha$ regulates tissue resident macrophage development}

The robust and dynamic expression of IL-7R by developing macrophages themselves suggested that IL-7R regulates fetal trMac development from precursors within fetal tissues. To test this hypothesis, we injected the highly specific IL-7R $\alpha$ monoclonal blocking antibody, A7R34, or an IgG2A control, into pregnant mice during fetal development (Fig. 6A). Injection of A7R34 during pregnancy completely blocks IL-7R signaling, as evidenced by deletion of Peyer's patches 
in developing embryos following a single injection (Hashizume et al., 2008; Yoshida et al., 1999). We injected pregnant WT mice with A7R34 or IgG2A control at E13.5 and E15.5, coincident with robust IL-7R $\alpha$ expression on fetal macrophages (Fig. 4B), and examined cellularity of macrophages in the epidermis, lung, liver, and brain in neonates 9 days later (Fig. 6A). Macrophage cellularity in the epidermis, liver, and lung was significantly reduced following this temporally-limited blockade of IL-7R $\alpha$ signaling, whereas microglia were unchanged (Fig. 6B). Coincidentally, limited IL-7R blockade resulted in a trend towards increased $\mathrm{CD} 11 \mathrm{~b}^{\mathrm{hi}} \mathrm{F} 4 / 80^{\mathrm{lo}}$ monocytes in the fetal liver $(\mathrm{p}=0.07)$ as well as a significant reduction in F4/80 ${ }^{\text {lo }}$ monocytes in the lung $(\mathrm{p}<0.05)$. These data provide additional evidence that IL-7R is regulating the transition from $\mathrm{F} 4 / 80^{\mathrm{lo}}$ macrophage precursor to $\mathrm{F} 4 / 80^{\mathrm{hi}}$ macrophage in peripheral tissues.

We further investigated trMac cellularity in IL $7 \mathrm{R}^{-/-}$mice, which have a germline deletion of IL7R $\alpha$ (Peschon et al., 1994). Although IL-7R deletion did not affect YS hematopoiesis (Fig. 2) examination at P19 revealed that IL-7R deletion drastically reduced cellularity of trMacs in all tissues (Fig. 6C). The most severe reductions were observed in the lung and epidermis (Fig. 6C), consistent with macrophages in those tissues expressing the highest and most sustained levels of IL-7R across development. Together with the IL-7R blocking experiments, these data indicate that IL-7R plays a unique role in the establishment of trMacs during perinatal development.

\section{Discussion}

Our investigation has revealed a novel role for IL-7R in fetal myeloid development, and specifically in the generation of fetal-specified trMacs. Tracing the progeny of cells mapped by the lymphoid marker IL-7R $\alpha$ revealed that trMacs derived from fetal hematopoiesis were distinctly marked by IL-7R $\alpha$ expression history (Fig. 1). In adult BM hematopoiesis, IL-7R $\alpha$ expression exclusively marks lymphoid cells (Schlenner et al., 2010), and the many functions of IL-7R signaling in lymphopoiesis are well-delineated (Fry and Mackall, 2005). We confirmed that adult trMacs do not express IL-7R $\alpha$ message or protein (Fig. 1H,I), and that both YS EMPs and adult BM-derived myeloid cells are minimally labeled by IL7R-Cre (Fig. 1C, 2A, S1). Instead, our developmental analysis revealed that labeling of trMacs by IL7R-Cre occurred as a 
result of dynamic stage- and tissue-specific expression of IL-7R during macrophage development within fetal tissues (Figs. 3,4). We showed that the CD11b ${ }^{\text {hi }}$ Ly6c ${ }^{\text {hi }}$ fetal liver monocytes rapidly upregulated surface IL-7R as they differentiated into F4/80 ${ }^{\text {hi }}$ macrophages ex vivo (Fig. 5). Using two different loss-of-function approaches, we further demonstrated that blocking IL-7R signaling during the window of robust IL7R expression by developing trMacs impairs their establishment (Fig. 6). By revealing a role for IL7R in trMac development, our data define a new signaling mechanism regulating trMac establishment in late gestation and identify a function of IL7R during fetal development that extends beyond regulation of the lymphoid lineage.

The comparison of trMac labeling across three different lineage tracing models based on lymphoid markers associated with both increasingly restricted lymphoid potential (Flk2, Rag1, IL-7R $\alpha$; Fig. 1) (Adolfsson et al., 2005; Forsberg et al., 2006; Igarashi et al., 2002; Kondo et al., 1997) as well as overlapping expression in FL progenitors (Beaudin et al., 2016; Boiers et al., 2013) allowed us to exclude the possibility that labeling of adult trMacs by IL7R-Cre reflected inheritance from an earlier lymphomyeloid progenitor. A clear example is epidermal langerhans cells: if labeling of LC was due to derivation from a lymphomyeloid progenitors, LC would be robustly labeled not only by IL7R-Cre, but also by Flk2-Cre and Rag1-Cre, as those genes are expressed in IL7R+ FL progenitors (Boiers et al., 2013). The high degree of IL7R-Cre labeling of trMacs was similarly not reflected in YS EMPs or YS macrophages (Fig. 2, Fig S2) and embryonic YS EMPs did not express significant IL-7R $\alpha$ message nor IL-7R surface protein. Instead, our tracking of IL7R-Cre mediated labeling (Fig. 3), IL-7R $\alpha$ mRNA levels (Fig. 4A) and IL-7R $\alpha$ surface expression (Fig. 4C), revealed dynamic regulation of IL-7R $\alpha$ expression by developing macrophage precursors and macrophages themselves during later stages of fetal hematopoiesis. Furthermore, blocking IL-7R during this limited window of development (E13.5-E15.5), when IL-7R was robustly expressed by fetal macrophages (Fig. 4D), impaired establishment of those populations (Fig. 6B). In contrast, IL-7R deletion had no effect on YS EMP cellularity or the development of YS macrophages during embryonic hematopoiesis (Fig. 2D, E), but resulted in significant impairments later in trMac development (Fig. 6C). Together, these data suggest that IL7R expression is important for the development of macrophages within 
tissues later in gestation, possibly during later waves of macrophage seeding (De et al., 2018; Ferrero et al., 2018; Tan and Krasnow, 2016).

Fetal F4/80 ${ }^{\text {hi }}$ macrophages expressed negligible IL-7R $\alpha$ message levels (Fig. 4A) yet expressed robust surface IL7R protein (Fig. 4D) and acquired IL7R-Cre labeling across fetal development (Fig. 3C). In lung and liver, for example, increased GFP labeling in F4/80 hi trMacs across fetal development did not reflect Cre-driven reporter expression, as trMacs did not express IL-7R $\alpha$ message during fetal development (Fig. 4A). The most parsimonious explanation for increased labeling is the initiation of Cre recombination in IL7R-marked F4/80 $1 \mathrm{lo}$ macrophage precursors with subsequent inheritance of the floxed allele by F4/80 ${ }^{\text {hi }}$ trMacs upon differentiation. F4/80 ${ }^{\text {hi }}$ trMac differentiation is then coincident with the rapid translation of IL-7R $\alpha$ message into IL-7R $\alpha$ protein and surface display (Fig. 6D). Indeed, our ex vivo differentiation assay revealed that Ly6c+ FL monocytes expressing IL-7R $\alpha$ mRNA (Fig. 4C; Fig. S3D) differentiate into F4/80 hi macrophages that upregulate surface IL-7R protein (Fig. 5). By tracking GFP expression within fetal myeloid cells in the IL7R-Cre model, we observed the contribution or replacement of GFPlabeled F4/80 $0^{\mathrm{lo}}$ precursors to F4/80 ${ }^{\mathrm{hi}}$ trMacs in the lung and liver, as evidenced by increased labeling in $\mathrm{F} 4 / 80^{\text {hi }}$ macrophages across ontogeny (Fig. 3C). Whereas trMacs of the lung and liver exhibited robust labeling ( 40\%) that had plateaued at birth (Fig. 3A-C), IL-7R $\alpha$ mRNA expression (Fig. 4B, D; (Mass et al., 2016)) and Cre-mediated labeling of LC and microglia continued postnatally, leading to almost complete labeling by IL7R-Cre in adulthood. In vivo, the rapid and dynamic regulation of IL-7R protein surface expression may reflect a response to ligand in tissues, as surface expression of IL-7R is regulated by IL-7 availability (Clark et al., 2014; Wei et al., 2000). The lower surface expression of IL-7R on fetal liver F4/80 ${ }^{\text {hi }}$ macrophages as compared to peripheral tissues (Fig. 4D) in vivo may also reflect surface regulation by immediate sources of IL-7 ligand in the fetal liver. Together, our analysis provides additional support for the contribution of FL F4/80 ${ }^{\text {lo }}$ macrophage precursors to specific trMac compartments during late gestation (Guilliams et al., 2013; Hoeffel et al., 2015; Rantakari et al., 2016; Tan and Krasnow, 2016), and reveal dynamic regulation of IL7R expression during macrophage differentiation. 
Our analysis of tissue macrophage development in the IL7R KO mouse and in response to physiological blockade of IL-7R reveal IL-7R as a novel regulator of tissue macrophage development. Blocking IL-7R $\alpha$ function during a narrow fetal window (E13.5 - E15.5) negatively affected cellularity of liver, lung, and epidermal macrophages, with no effect on microglia. In contrast, germline deletion of IL-7R $\alpha$ in the IL $7 \mathrm{R}^{-/-}$mouse significantly impaired trMac cellularity across all tissues, including microglia, examined at postnatal day 19 (Fig. 6C). The different effect of total knockout and transient blockade of IL-7R $\alpha$ on trMac development suggests that the window of dependency on IL7R signaling differs between different tissues. Furthermore, the effect of transient blockade of IL7R signaling at a stage when fetal macrophages themselves express robust surface levels of IL7R (e.g. late gestation) suggests that IL 7R is directly regulating macrophage development in peripheral tissues. In contrast, deletion of IL7R had no effect on YS EMPs or YS macrophage generation, affirming that IL7R plays a more critical role in fetal macrophage generation at later stages of hematopoiesis. Regardless of whether IL7R+ fetal progenitors contribute to the trMac pool, the uniform and robust IL7R surface expression on fetal trMac cells themselves was highly surprising, and contributes to accumulating evidence that fetal hematopoietic lineage specification is considerably less constrained than adult hematopoiesis (Beaudin et al., 2016; Mebius et al., 2001; Notta et al., 2016).

\section{Methods}

Mice

All animals were housed and bred in the AALAC accredited vivaria at UC Santa Cruz or UC Merced and group housed in ventilated cages on a standard 12:12 light cycle. All procedures were approved by the UCSC or the UC Merced Institutional Animal Care and Use (IACUC) committees. IL7R $\alpha$-Cre (Schlenner et al., 2010), Rag1-Cre (Welner et al., 2009), and Flk2-Cre (Benz et al., 2008) mice, obtained under fully executed Material Transfer Agreements, were crossed to homozygous Rosa $26^{\mathrm{mTmG}}$ females (Muzumdar et al., 2007) to generate "switch" lines, all on the $\mathrm{C} 57 \mathrm{Bl} / 6$ background. WT C56B1/6 mice were used for controls and for all expression experiments. Adult male and female mice were used randomly and indiscriminately, with the exception of the FlkSwitch line, in which only males were used because many female mice do 
not carry a Cre allele. Similarly, mice for developmental analysis were used indiscriminately without knowledge of gender.

\section{Tissue and cell isolation}

Mice were sacrificed by $\mathrm{CO}_{2}$ inhalation. Gravid uteri removed, and individual embryos dissected. Adult liver, lung, brain, and skin were isolated and treated with $1 \times \mathrm{PBS}(+/+)$ with $2 \%$ serum, $0.2-1 \mathrm{mg} / \mathrm{ml}$ collagenase IV (Gibco) with or without 100U/ml DNase1 for 20 minutes to 2 hours. For adult epidermis isolation, ears were first incubated with 1X PBS $(+/+)$ containing $2.4 \mathrm{mg} / \mathrm{ml}$ dispase (Gibco) to separate the epidermis, followed by 2 hours incubation of the epidermis with $1 / \mathrm{mg} / \mathrm{mL}$ collagenase. Following incubation, all tissues were passaged through a $16 \mathrm{~g}$ needle or a $19 \mathrm{~g}$ needle $10 \mathrm{X}$, and then filtered through a $70 \mu \mathrm{M}$ filter.

\section{Flow Cytometry}

Cell labeling was performed on ice in $1 \mathrm{X}$ PBS with $5 \mathrm{mM}$ EDTA and 2\% serum. Antibodies used are listed in the supplementary data table 1. Analysis was performed on BD FACS Aria II at University of California-Santa Cruz, and BD FACS Aria III and the University of CaliforniaMerced and analyzed using FlowJo.

\section{Transplantation Assays}

Transplantation assays were performed as previously described. (Beaudin et al., 2014; Beaudin et al., 2016; Smith-Berdan et al., 2015; Ugarte et al., 2015) Briefly, sorted Tom+ or GFP+ KLS cells were isolated from IL7R $\alpha$ Switch or Rag1Switch E14.5 fetal liver donors. WT recipient mice aged 8-12 weeks were sublethally iradiated (750 rad, single dose). Under isofluoraneinduced general anesthesia, sorted cells were transplanted IV. Recipient mice were bled 4, 8, 12, and 16 weeks post transplantation via tail vein and peripheral blood was analyzed for donor chimerism by means of fluorescence profiles and antibodies to lineage markers. Long-term multilineage reconstitution was defined as chimerism in both the lymphoid and myeloid lineages of $>0.1 \%$ at 16 weeks post-transplantation. 


\section{Ex vivo macrophage culture}

E14.5 fetal livers from WT embryos were harvested and homogenized via trituration. CD11b+ cells were enriched by positive selection with CD11b biotin-conjugated antibodies and streptavidin microbeads, using LS columns (Miltenyi). F4/80 ${ }^{\text {lo }} \mathrm{CD} 11 \mathrm{~b}^{\text {hi }}$ cells were sorted based on Ly6c expression and cultured in a 96-well plate (DMEM, 20\% FBS, 1 mM Sodium Pyruvate, $10 \mathrm{mM}$ HEPES, $0.1 \mathrm{mM}$ 2-Mercaptoethanol, and $50 \mathrm{mg} / \mathrm{mL}$ primocin, Life technologies). Cells were cultured in triplicate in the presence of $20 \mathrm{ng} / \mathrm{mL}$ M-CSF and analyzed at three different time points (Days 1, 3, and 5) for the presence of F4/80 ${ }^{\text {hi }} \mathrm{CD} 11 \mathrm{~b}+$ macrophages and for surface expression of IL7R, common gamma chain (CD132), and CD64.

RNA Isolation and Quantitative Real-time Polymerase Chain Reaction (qRT-PCR) Analysis: RNA isolation from sorted cells was accomplished using the RNeasy mini kit (Qiagen, Hilden, Germany), and cDNA was reverse transcribed from RNA (High capacity cDNA reverse transcription kit, ThermoFisher Scientific, Massachussetts). TaqMan probes (TaqMan Gene Expression Assay, ThermoFisher Scientific) were used for qRT-PCR analysis on a StepOnePlus Real-Time PCR System (ThermoFisher Scientific) in comparative CT mode. Samples were run in triplicate and were run with positive (CD43+ B220+ Pro B-cells), negative (Gr1+ CD11b+ BM monocytes), and no cDNA controls.

\section{Quantification and Statistical Analysis}

Number of experiments, $\mathrm{n}$, and what $\mathrm{n}$ represents can be found in the legend for each figure. Statistical significance was determined by two-tailed unpaired student's T-test. All data are shown as mean \pm standard error of the mean (SEM) representing at least three independent experiments. 


\section{References:}

Adolfsson, J., R. Mansson, N. Buza-Vidas, A. Hultquist, K. Liuba, C.T. Jensen, D. Bryder, L. Yang, O.J. Borge, L.A. Thoren, K. Anderson, E. Sitnicka, Y. Sasaki, M. Sigvardsson, and S.E. Jacobsen. 2005. Identification of Flt3+ lympho-myeloid stem cells lacking erythromegakaryocytic potential a revised road map for adult blood lineage commitment. Cell 121:295306.

Ajami, B., J.L. Bennett, C. Krieger, W. Tetzlaff, and F.M. Rossi. 2007. Local self-renewal can sustain CNS microglia maintenance and function throughout adult life. Nat Neurosci 10:15381543.

Beaudin, A.E., S.W. Boyer, and E.C. Forsberg. 2014. Flk2/Flt3 promotes both myeloid and lymphoid development by expanding non-self-renewing multipotent hematopoietic progenitor cells. Exp Hematol 42:218-229 e214.

Beaudin, A.E., S.W. Boyer, J. Perez-Cunningham, G.E. Hernandez, S.C. Derderian, C. Jujjavarapu, E. Aaserude, T. MacKenzie, and E.C. Forsberg. 2016. A Transient Developmental Hematopoietic Stem Cell Gives Rise to Innate-like B and T Cells. Cell Stem Cell 19:768-783.

Beaudin, A.E., and E.C. Forsberg. 2016. To B1a or not to B1a: do hematopoietic stem cells contribute to tissue-resident immune cells? Blood 128:2765-2769.

Benz, C., V.C. Martins, F. Radtke, and C.C. Bleul. 2008. The stream of precursors that colonizes the thymus proceeds selectively through the early $\mathrm{T}$ lineage precursor stage of $\mathrm{T}$ cell development. J Exp Med 205:1187-1199.

Boiers, C., J. Carrelha, M. Lutteropp, S. Luc, J.C. Green, E. Azzoni, P.S. Woll, A.J. Mead, A. Hultquist, G. Swiers, E.G. Perdiguero, I.C. Macaulay, L. Melchiori, T.C. Luis, S. Kharazi, T. Bouriez-Jones, Q. Deng, A. Ponten, D. Atkinson, C.T. Jensen, E. Sitnicka, F. Geissmann, I. Godin, R. Sandberg, M.F. de Bruijn, and S.E. Jacobsen. 2013. Lymphomyeloid Contribution of 
an Immune-Restricted Progenitor Emerging Prior to Definitive Hematopoietic Stem Cells. Cell Stem Cell

Boyer, S.W., A.E. Beaudin, and E.C. Forsberg. 2012. Mapping stem cell differentiation pathways from hematopoietic stem cells using Flk2/Flt3L lineage tracing. Cell Cycle 11:31803188.

Boyer, S.W., A.V. Schroeder, S. Smith-Berdan, and E.C. Forsberg. 2011. All hematopoietic cells develop from hematopoietic stem cells through Flk2/Flt3-positive progenitor cells. Cell Stem Cell 9:64-73.

Clark, M.R., M. Mandal, K. Ochiai, and H. Singh. 2014. Orchestrating B cell lymphopoiesis through interplay of IL-7 receptor and pre-B cell receptor signalling. Nat Rev Immunol 14:69-80.

Cool, T., and E.C. Forsberg. 2019. Chasing Mavericks: The quest for defining developmental waves of hematopoiesis. Current Topics in Developmental Biology. In press.

De, S., D. Van Deren, E. Peden, M. Hockin, A. Boulet, S. Titen, and M.R. Capecchi. 2018. Two distinct ontogenies confer heterogeneity to mouse brain microglia. Development 145 :

Epelman, S., K.J. Lavine, A.E. Beaudin, D.K. Sojka, J.A. Carrero, B. Calderon, T. Brija, E.L. Gautier, S. Ivanov, A.T. Satpathy, J.D. Schilling, R. Schwendener, I. Sergin, B. Razani, E.C. Forsberg, W.M. Yokoyama, E.R. Unanue, M. Colonna, G.J. Randolph, and D.L. Mann. 2014. Embryonic and adult-derived resident cardiac macrophages are maintained through distinct mechanisms at steady state and during inflammation. Immunity 40:91-104.

Ferrero, G., C.B. Mahony, E. Dupuis, L. Yvernogeau, E. Di Ruggiero, M. Miserocchi, M. Caron, C. Robin, D. Traver, J.Y. Bertrand, and V. Wittamer. 2018. Embryonic Microglia Derive from Primitive Macrophages and Are Replaced by cmyb-Dependent Definitive Microglia in Zebrafish. Cell Rep 24:130-141. 
Forsberg, E.C., T. Serwold, S. Kogan, I.L. Weissman, and E. Passegue. 2006. New evidence supporting megakaryocyte-erythrocyte potential of flk2/flt3+ multipotent hematopoietic progenitors. Cell 126:415-426.

Fry, T.J., and C.L. Mackall. 2005. The many faces of IL-7: from lymphopoiesis to peripheral T cell maintenance. J Immunol 174:6571-6576.

Ginhoux, F., M. Greter, M. Leboeuf, S. Nandi, P. See, S. Gokhan, M.F. Mehler, S.J. Conway, L.G. Ng, E.R. Stanley, I.M. Samokhvalov, and M. Merad. 2010. Fate mapping analysis reveals that adult microglia derive from primitive macrophages. Science 330:841-845.

Gomez Perdiguero, E., K. Klapproth, C. Schulz, K. Busch, E. Azzoni, L. Crozet, H. Garner, C. Trouillet, M.F. de Bruijn, F. Geissmann, and H.R. Rodewald. 2015. Tissue-resident macrophages originate from yolk-sac-derived erythro-myeloid progenitors. Nature 518:547-551.

Guilliams, M., I. De Kleer, S. Henri, S. Post, L. Vanhoutte, S. De Prijck, K. Deswarte, B. Malissen, H. Hammad, and B.N. Lambrecht. 2013. Alveolar macrophages develop from fetal monocytes that differentiate into long-lived cells in the first week of life via GM-CSF. J Exp Med 210:1977-1992.

Hashimoto, D., A. Chow, C. Noizat, P. Teo, M.B. Beasley, M. Leboeuf, C.D. Becker, P. See, J. Price, D. Lucas, M. Greter, A. Mortha, S.W. Boyer, E.C. Forsberg, M. Tanaka, N. van Rooijen, A. Garcia-Sastre, E.R. Stanley, F. Ginhoux, P.S. Frenette, and M. Merad. 2013. Tissue-resident macrophages self-maintain locally throughout adult life with minimal contribution from circulating monocytes. Immunity 38:792-804.

Hashizume, T., A. Togawa, T. Nochi, O. Igarashi, M.N. Kweon, H. Kiyono, and M. Yamamoto. 2008. Peyer's patches are required for intestinal immunoglobulin A responses to Salmonella spp. Infect Immun 76:927-934.

Hoeffel, G., J. Chen, Y. Lavin, D. Low, F.F. Almeida, P. See, A.E. Beaudin, J. Lum, I. Low, E.C. Forsberg, M. Poidinger, F. Zolezzi, A. Larbi, L.G. Ng, J.K. Chan, M. Greter, B. Becher, 
I.M. Samokhvalov, M. Merad, and F. Ginhoux. 2015. C-myb(+) erythro-myeloid progenitorderived fetal monocytes give rise to adult tissue-resident macrophages. Immunity 42:665-678.

Hoeffel, G., Y. Wang, M. Greter, P. See, P. Teo, B. Malleret, M. Leboeuf, D. Low, G. Oller, F. Almeida, S.H. Choy, M. Grisotto, L. Renia, S.J. Conway, E.R. Stanley, J.K. Chan, L.G. Ng, I.M. Samokhvalov, M. Merad, and F. Ginhoux. 2012. Adult Langerhans cells derive predominantly from embryonic fetal liver monocytes with a minor contribution of yolk sac-derived macrophages. J Exp Med 209:1167-1181.

Hulsmans, M., S. Clauss, L. Xiao, A.D. Aguirre, K.R. King, A. Hanley, W.J. Hucker, E.M. Wülfers, G. Seemann, G. Courties, Y. Iwamoto, Y. Sun, A.J. Savol, H.B. Sager, K.J. Lavine, G.A. Fishbein, D.E. Capen, N. Da Silva, L. Miquerol, H. Wakimoto, C.E. Seidman, J.G.

Seidman, R.I. Sadreyev, K. Naxerova, R.N. Mitchell, D. Brown, P. Libby, R. Weissleder, F.K. Swirski, P. Kohl, C. Vinegoni, D.J. Milan, P.T. Ellinor, and M. Nahrendorf. 2017. Macrophages Facilitate Electrical Conduction in the Heart. Cell 169:510-522.e520.

Igarashi, H., S.C. Gregory, T. Yokota, N. Sakaguchi, and P.W. Kincade. 2002. Transcription from the RAG1 locus marks the earliest lymphocyte progenitors in bone marrow. Immunity 17:117-130.

Kierdorf, K., D. Erny, T. Goldmann, V. Sander, C. Schulz, E.G. Perdiguero, P. Wieghofer, A. Heinrich, P. Riemke, C. Hölscher, D.N. Müller, B. Luckow, T. Brocker, K. Debowski, G. Fritz, G. Opdenakker, A. Diefenbach, K. Biber, M. Heikenwalder, F. Geissmann, F. Rosenbauer, and M. Prinz. 2013. Microglia emerge from erythromyeloid precursors via Pu.1- and Irf8-dependent pathways. Nat Neurosci 16:273-280.

Kondo, M., I.L. Weissman, and K. Akashi. 1997. Identification of clonogenic common lymphoid progenitors in mouse bone marrow. Cell 91:661-672.

Mass, E., I. Ballesteros, M. Farlik, F. Halbritter, P. Günther, L. Crozet, C.E. Jacome-Galarza, K. Händler, J. Klughammer, Y. Kobayashi, E. Gomez-Perdiguero, J.L. Schultze, M. Beyer, C. 
Bock, and F. Geissmann. 2016. Specification of tissue-resident macrophages during organogenesis. Science 353:

Mebius, R.E., T. Miyamoto, J. Christensen, J. Domen, T. Cupedo, I.L. Weissman, and K. Akashi. 2001. The fetal liver counterpart of adult common lymphoid progenitors gives rise to all lymphoid lineages, CD45+CD4+CD3- cells, as well as macrophages. Journal of immunology 166:6593-6601.

Merad, M., M.G. Manz, H. Karsunky, A. Wagers, W. Peters, I. Charo, I.L. Weissman, J.G. Cyster, and E.G. Engleman. 2002. Langerhans cells renew in the skin throughout life under steady-state conditions. Nat Immunol 3:1135-1141.

Muzumdar, M.D., B. Tasic, K. Miyamichi, L. Li, and L. Luo. 2007. A global double-fluorescent Cre reporter mouse. Genesis 45:593-605.

Notta, F., S. Zandi, N. Takayama, S. Dobson, O.I. Gan, G. Wilson, K.B. Kaufmann, J. McLeod, E. Laurenti, C.F. Dunant, J.D. McPherson, L.D. Stein, Y. Dror, and J.E. Dick. 2016. Distinct routes of lineage development reshape the human blood hierarchy across ontogeny. Science 351:aab2116.

Peschon, J.J., P.J. Morrissey, K.H. Grabstein, F.J. Ramsdell, E. Maraskovsky, B.C. Gliniak, L.S. Park, S.F. Ziegler, D.E. Williams, C.B. Ware, J.D. Meyer, and B.L. Davison. 1994. Early lymphocyte expansion is severely impaired in interleukin 7 receptor-deficient mice. $J$ Exp Med 180:1955-1960.

Rantakari, P., N. Jäppinen, E. Lokka, E. Mokkala, H. Gerke, E. Peuhu, J. Ivaska, K. Elima, K. Auvinen, and M. Salmi. 2016. Fetal liver endothelium regulates the seeding of tissue-resident macrophages. Nature 538:392-396.

Sawai, C.M., S. Babovic, S. Upadhaya, D.J. Knapp, Y. Lavin, C.M. Lau, A. Goloborodko, J. Feng, J. Fujisaki, L. Ding, L.A. Mirny, M. Merad, C.J. Eaves, and B. Reizis. 2016. 
Hematopoietic Stem Cells Are the Major Source of Multilineage Hematopoiesis in Adult Animals. Immunity 45:597-609.

Schlenner, S.M., V. Madan, K. Busch, A. Tietz, C. Laufle, C. Costa, C. Blum, H.J. Fehling, and H.R. Rodewald. 2010. Fate mapping reveals separate origins of T cells and myeloid lineages in the thymus. Immunity 32:426-436.

Schulz, C., E. Gomez Perdiguero, L. Chorro, H. Szabo-Rogers, N. Cagnard, K. Kierdorf, M. Prinz, B. Wu, S.E. Jacobsen, J.W. Pollard, J. Frampton, K.J. Liu, and F. Geissmann. 2012. A lineage of myeloid cells independent of Myb and hematopoietic stem cells. Science 336:86-90.

Sheng, J., C. Ruedl, and K. Karjalainen. 2015. Most Tissue-Resident Macrophages Except Microglia Are Derived from Fetal Hematopoietic Stem Cells. Immunity 43:382-393.

Smith-Berdan, S., A. Nguyen, M.A. Hong, and E.C. Forsberg. 2015. ROBO4-mediated vascular integrity regulates the directionality of hematopoietic stem cell trafficking. Stem Cell Reports $4: 255-268$.

Stremmel, C., R. Schuchert, F. Wagner, R. Thaler, T. Weinberger, R. Pick, E. Mass, H.C. Ishikawa-Ankerhold, A. Margraf, S. Hutter, R. Vagnozzi, S. Klapproth, J. Frampton, S. Yona, C. Scheiermann, J.D. Molkentin, U. Jeschke, M. Moser, M. Sperandio, S. Massberg, F. Geissmann, and C. Schulz. 2018. Yolk sac macrophage progenitors traffic to the embryo during defined stages of development. Nat Commun 9:75.

Tan, S.Y., and M.A. Krasnow. 2016. Developmental origin of lung macrophage diversity. Development 143:1318-1327.

Ugarte, F., R. Sousae, B. Cinquin, E.W. Martin, J. Krietsch, G. Sanchez, M. Inman, H. Tsang, M. Warr, E. Passegue, C.A. Larabell, and E.C. Forsberg. 2015. Progressive Chromatin Condensation and H3K9 Methylation Regulate the Differentiation of Embryonic and Hematopoietic Stem Cells. Stem Cell Reports 5:728-740. 
Wei, C., R. Zeff, and I. Goldschneider. 2000. Murine pro-B cells require IL-7 and its receptor complex to up-regulate IL-7R alpha, terminal deoxynucleotidyltransferase, and c mu expression. J Immunol 164:1961-1970.

Welner, R.S., B.L. Esplin, K.P. Garrett, R. Pelayo, H. Luche, H.J. Fehling, and P.W. Kincade. 2009. Asynchronous RAG-1 expression during B lymphopoiesis. J Immunol 183:7768-7777.

Yona, S., K.W. Kim, Y. Wolf, A. Mildner, D. Varol, M. Breker, D. Strauss-Ayali, S. Viukov, M. Guilliams, A. Misharin, D.A. Hume, H. Perlman, B. Malissen, E. Zelzer, and S. Jung. 2013. Fate mapping reveals origins and dynamics of monocytes and tissue macrophages under homeostasis. Immunity 38:79-91.

Yoshida, H., K. Honda, R. Shinkura, S. Adachi, S. Nishikawa, K. Maki, K. Ikuta, and S.I. Nishikawa. 1999. IL-7 receptor alpha+ CD3(-) cells in the embryonic intestine induces the organizing center of Peyer's patches. Int Immunol 11:643-655. 
Acknowledgments: We thank Drs. Hans-Reimer Rodewald and Susan M. Schlenner for the IL 7R $\alpha^{\text {Cre }}$ strain; Drs. Patricia Ernst and Terri Rabbitt for the Rag1 ${ }^{\text {Cre }}$ strain; Dr T. Boehm for the Flt $3^{\mathrm{Cre}}$ strain; Bari Nazario and the UCSC Institute for the Biology of Stem Cells for flow cytometry support; and David Gravano and the UC Merced Stem Cell Instrumentation Foundry for flow cytometry support. Funding: A.E.B. is the recipient of an NHLBI Mentored Career Development Award (K01HL130753). This work was supported by an NIH/NIDDK award (R01DK100917), an Alex's Lemonade Stand Foundation Innovation Award, and an American Asthma Foundation Research Scholar award to ECF; by CIRM SCILL grant TB1-01195 to TM via San Jose State University; and by CIRM Facilities awards CL1-00506 and FA1-00617-1 to UCSC. ECF is the recipient of a CIRM New Faculty Award (RN1-00540) and an American Cancer Society Research Scholar Award (RSG-13-193-01-DDC). Author contributions: A.E.B. and E.C.F. conceived of the study, designed the experiments, and co-wrote the paper. A.E.B., T.M., G.L., A.W., and C.V. performed experiments and analyzed data. All authors reviewed the manuscript.

Competing interests: The authors have no conflicting financial interests. 


\section{Figure Legends}

\section{Figure 1. IL7R-Cre specifically labels adult tissue-resident macrophage populations.}

Representative flow cytometric analysis of reporter expression across different monocyte and macrophage populations in adult mice. Tom and GFP expression is highlighted by red and green boxes, respectively, in FlkSwitch, Rag1Switch, and IL7Rswitch models. Values indicate mean frequencies \pm SEM of gated IL7R-Cre marked GFP+ populations. Plots and values are representative of 4-5 mice each representing three independent experiments.

A, Schematic of the "Switch" models. Cre recombinase expression was controlled by either Flk2, IL7R $\alpha$, or Rag1 regulatory elements, respectively. Cre-driver mice were crossed to mice expressing a dual color reporter expressing either Tomato (Tom) or GFP, under control of the Rosa26 locus. Expression of Cre results in an irreversible genetic deletion event that causes a switch in reporter expression from Tomato to GFP.

B, Schematic of Cre-mediated reporter switching in the "switch" models. All cells initially express Tomato. Expression of Cre results in an irreversible switch from Tomato to GFP expression. Once a cell expresses GFP, it can only give rise to GFP-expressing progeny. C, Representative flow cytometric analysis of reporter expression in circulating $\mathrm{CD} 11 \mathrm{~b}^{\mathrm{hi}} \mathrm{Gr}^{\mathrm{mid}}$ monocytes in the peripheral blood (PB) of adult FlkSwitch, Rag1Switch, and IL7RSwitch mice. D, Representative flow cytometric analysis of reporter expression in Langerhans cells $\left(\mathrm{F} 4 / 80+\mathrm{CD} 11 \mathrm{~b}+\mathrm{CD} 11 \mathrm{c}^{\mathrm{mid}}\right)$ in the epidermis of adult FlkSwitch, Rag1Switch, and IL7RSwitch mice.

E, Representative flow cytometric analysis of reporter expression in microglia

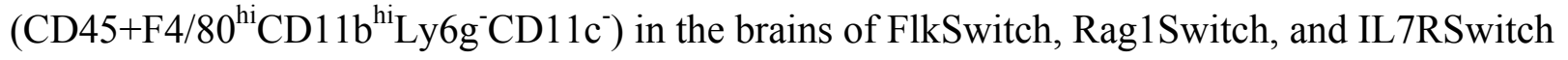
adult mice.

F, Representative flow cytometric analysis of reporter expression in lung alveolar macrophages $\left(\mathrm{CD} 45+\mathrm{F} 4 / 80^{\mathrm{hi}} \mathrm{CD} 11 \mathrm{~b}^{\mathrm{mid}}\right.$ SiglecF $\left.^{+} \mathrm{CD} 11 \mathrm{c}^{+}\right)$of FlkSwitch, Rag1Switch, and IL7RSwitch adult mice.

G, Representative flow cytometric analysis of reporter expression in Liver Kupffer cells $\left(\mathrm{CD} 45^{+} \mathrm{F} 4 / 80^{\mathrm{hi}} \mathrm{CD} 169^{+}\right)$in adult FlkSwitch, Rag1Switch, and IL7RSwitch mice.

H, Lack of IL-7R $\alpha$ surface expression in the Langerhans cells of the epidermis, brain microglia, lung alveolar macrophages, and liver Kupffer cells. For each tissue, IL-7R $\alpha$ surface expression 
of gated population is shown for two representative mice in blue. Gray shaded area represents fluorescence-minus-one (FMO) control.

I, Quantitative RT-PCR analysis of IL-7R $\alpha$ expression in sorted bone marrow (BM)-derived B220+CD43+ ProB-cells and CD11b+Gr1+monocytes, and Langerhans cells of the epidermis, brain microglia, lung alveolar macrophages, and Liver Kupffer cells isolated from WT adult mice. Data represent mean \pm SEM for 3 independent experiments. Values are normalized to Pro-B cells, set to 100. ND, not detected. Additional analyses of adult tissue myeloid populations can be found in Figure S1. 


\section{Figure 2. IL-7Ra is dispensable for YS hematopoiesis}

A, $\mathbf{A}^{\prime}$, Representative FACS plots showing gating strategy, IL7R-Cre-driven GFP labeling, and IL-7R $\alpha$ surface expression in Ter119-cKit+CD41+ erythromyeloid progenitors (EMPs) in the yolk sac (YS) at E9.5, E10.5, E11.5 and in the aorta-gonad-mesonephros region (AGM) at E11.5. Red and green boxes denote gates for Tom+ and GFP+ progenitors, respectively. Gray shaded areas in histograms indicate FMO control. Plots and values indicate mean frequencies \pm SEM of gated IL7R-Cre marked GFP+ populations for 4-7 mice from at least two independent experiments.

B, Representative FACS plots showing gating, IL7R-Cre-driven GFP labeling, and IL7R $\alpha$ surface expression in CD45+Ter119- cells in the YS at E9.5. Adjacent plot shows CD11b and F4/80 expression within the CD45+Ter119- compartment. Red and green boxes denote gates for Tom and GFP expression of CD45+Ter119- cells, respectively.

C, $\mathbf{C}^{\prime}$, Quantitative RT-PCR analysis of IL-7R $\alpha$ mRNA in YS EMPs from E9.5. (C) $\Delta \Delta C^{\mathrm{T}}$ values normalized to beta-Actin as compared to IL-7R $\alpha$ expression levels in MDPs (CD115+cKit+Flk2+Ly6c-) isolated from E14.5 FL. (C') Representative amplification plot of beta-actin (solid) and IL-7R $\alpha$ (dashed) for quantitative RT-PCR of E14.5 MDPs and EMPs as described in $(\mathrm{C})$.

Data show mean \pm SEM from 3 independent experiments.

D, E, Quantification of the frequency of EMPs (D) and F4/80+ macrophages (E) in YS of

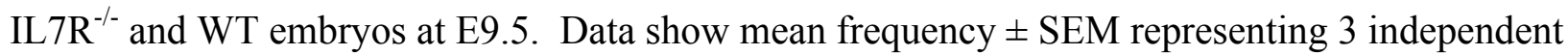
experiments.

F,G, Representative FACS plots showing gating, IL7R-Cre-driven GFP labeling, and IL-7R $\alpha$ surface expression in cKit+Lin-Sca1+ stem and progenitor cells at E12.5 (F) and E14.5 (G).

H, Quantification across development of the percent of IL 7R-Cre driven reporter labeling $(\mathrm{GFP}+)$ in the phenotypically defined stem and progenitor populations described in A-G. Additional analysis of IL7R-Cre driven reporter labeling in fetal hematopoietic populations can be found in Figure S2. 


\section{Figure 3. IL7R-Cre dynamically labels myeloid cells during tissue resident macrophage}

\section{development.}

A, Representative flow cytometric analysis indicating gating for fetal liver (FL)-derived F4/80 ${ }^{\text {lo }}$ monocytes $\left(\mathrm{CD} 45+\mathrm{F} 4 / 80^{\mathrm{lo}} \mathrm{CD} 11 \mathrm{~b}{ }^{\text {hi }}\right.$; orange gates) and previously seeded $\mathrm{F} 4 / 80^{\mathrm{hi}}$ macrophages $\left(\mathrm{CD} 45+\mathrm{F} 4 / 80^{\mathrm{hi}} \mathrm{CD} 11 \mathrm{~b}^{\mathrm{mid}}\right.$; purple gates) in different tissues at E12.5, E14.5 and P0.

B, Quantification of IL7R-Cre driven GFP labeling within gated population indicated in (A) across different tissues at E12.5, E14.5, P0 and Adult (8-12 weeks). Orange asterisks denote cross-tissue differences in GFP labeling between F4/80 ${ }^{\text {lo }}$ monocytes and purple asterisks denote cross-tissue differences in GFP labeling between F4/80 ${ }^{\text {hi }}$ macrophages. Black asterisks denote differences in GFP labeling between F4/80 ${ }^{\text {lo }}$ monocytes and $\mathrm{F} 4 / 80^{\mathrm{hi}}$ macrophages within each tissue. $\mathrm{N}=4-11$ representing at least 3 independent experiments. $* \mathrm{P}<0.05, * * \mathrm{P}<0.005, * * * \mathrm{P}$ $<0.0005$.

C, Time course of IL7R-Cre driven GFP labeling in F4/80 ${ }^{\mathrm{hi}}$ macrophages and F4/80 $0^{\mathrm{lo}}$ monocytes shown in (B) across ontogeny. Asterisks indicate statistically significant differences between the percentage of GFP labeling at the previously measured timepoint, with purple asterisks denoting differences between the indicated $\mathrm{F} 4 / 80^{\mathrm{hi}}$ macrophages and orange asterisks denoting differences between $\mathrm{F} 4 / 80^{\mathrm{lo}}$ monocyte populations.

Additional analysis of fetal myeloid populations can be found in Figure S3. 
Figure 4. IL-7Ra message and surface expression are dynamically regulated during macrophage development

A, Quantitative RT-PCR analysis of IL-7R $\alpha$ mRNA in monocytes $\left(F 4 / 80^{10} \mathrm{CD} 11 \mathrm{~b}^{\mathrm{hi}}\right)$ and macrophages $\left(\mathrm{F} 4 / 80^{\text {hi }} \mathrm{CD} 11 \mathrm{~b}^{\mathrm{lo}}\right)$ isolated from liver, lung, brain, and skin of E14.5 fetuses. Fetal liver macrophage dendritic cell precursors (MDP; CD115+cKit+Flk2+Ly6c-CD11b-) and adult BM monocytes (CD11b+Gr1mid) were used as positive and negative controls, respectively. Data shown are mean \pm standard error of $\Delta \Delta \mathrm{CT}$ values calculated for IL7R $\alpha$ and Actin and normalized to MDPs (set to 100) across three independent experiments.

B, Quantitative RT-PCR analysis of IL-7R $\alpha$ mRNA in monocytes (F4/80 $\left.{ }^{\mathrm{lo}} \mathrm{CD} 11 \mathrm{~b}^{\mathrm{hi}}\right)$ and macrophages $\left(\mathrm{F} 4 / 80^{\mathrm{hi}} \mathrm{CD} 11 \mathrm{~b}^{\mathrm{lo}}\right)$ isolated from liver, lung, brain, and epidermis of neonatal mice at P14. Adult BM ProB cells (B220+CD43+) and BM monocytes (CD11b+Gr1+) were used as positive and negative controls, respectively. Data shown are mean $\mp$ standard error of $\Delta \Delta \mathrm{CT}$ values calculated for IL-7R $\alpha$ and beta-actin and normalized to BM ProB cells (set to 100) across three independent experiments. ND, not detected

C,D Representative flow cytometric analysis of IL-7R $\alpha$ surface expression in F4/80 ${ }^{\mathrm{lo}}$ monocytes $\left(\mathrm{CD} 45+\mathrm{F} 4 / 80^{\mathrm{lo}} \mathrm{CD} 11 \mathrm{~b}^{\mathrm{hi}} ; \mathbf{C}\right)$ and $\mathrm{F} 4 / 80^{\mathrm{hi}}$ macrophages $\left(\mathrm{CD} 45+\mathrm{F} 4 / 80^{\mathrm{hi}} \mathrm{CD} 11 \mathrm{~b}^{\mathrm{mid}} ; \mathbf{D}\right)$ in different tissues at embryonic day (E)12.5, E14.5, E17.5, postnatal day (P)0 and P14. Gray shaded area indicates FMO control. Plots are representative of analysis in 5-6 mice from three independent experiments.

Analysis of common gamma chain expression in fetal tissue macrophages can be found in Figure S4. 


\section{Figure 5. Fetal liver monocytes differentiate into IL-7R-expressing macrophages ex vivo}

A, Representative gating of $\mathrm{F} 4 / 80^{\mathrm{lo}} \mathrm{CD} 11 \mathrm{~b}{ }^{\text {hi }}$ monocytes (orange gates) from $\mathrm{CD} 11 \mathrm{~b}$-enriched fetal liver (FL) at E14.5, gated on Ly6c expression (Ly6c ${ }^{\text {hi }}$, Red; Ly6c ${ }^{\text {lo }}$, Blue). Adjacent plots represent post-sort purity analysis of FL CD $11 b^{\text {hi }} \mathrm{F} 4 / 80^{\text {lo }}$ Ly $6 \mathrm{c}^{\text {hi }}$ and Ly $6 \mathrm{c}^{\text {lo }}$ monocytes with mean \pm SEM frequency of sorted populations in triplicate from 3 independent experiments.

B, Representative FACS plots show surface IL-7R $\alpha$ expression on macrophage (F4/80 ${ }^{\mathrm{hi}}$ ) and monocyte $\left(\mathrm{F} 4 / 80^{\mathrm{lo}} \mathrm{CD} 11 \mathrm{~b}^{\mathrm{hi}}\right)$ populations as a function of Ly6c expression, color-coded as gated in $(A)$.

C-E, Representative FACS plots showing gating of monocyte $\left(\mathrm{F} 4 / 80^{10} \mathrm{CD} 11 \mathrm{~b}+\right)$ and macrophage $\left(\mathrm{F} 4 / 80^{\mathrm{hi}} \mathrm{CD} 11 \mathrm{~b}+\right)$ populations and expression of IL-7R $\alpha(\mathbf{C}), \mathrm{CD} 132(\mathbf{D})$, and CD64 (E) on gated populations following 1,3 , or 5 days of culture of sorted $\mathrm{F} 4 / 80^{\mathrm{lo}} \mathrm{CD} 11 \mathrm{~b}^{\mathrm{hi}} \mathrm{Ly} 6 \mathrm{c}^{\mathrm{hi}}$ monocytes with 20 ng m-CSF. Mean fluorescence intensity (MFI; $\left.\mathbf{C}^{\prime}, \mathbf{D}^{\prime}, \mathbf{E}^{\prime}\right)$ was calculated as the geometric mean minus the FMO for each sample. Gray shaded area indicates FMO control. Values indicate mean frequencies \pm SEM of gated populations for 6-8 different samples in triplicate from 3 independent experiments. *. $\mathrm{P}<0.05, * * \mathrm{P}<0.01, * * * \mathrm{P}<0.001$, Student's T-test. 


\section{Figure 6. IL-7R regulates tissue resident macrophage development.}

A, Schematic illustrating the timeline for injection and analysis of the IL7R blocking antibody, A7R43, and IgG2A control. Timed-mated WT mice were injected at embryonic day (E)13.5 and E15.5 with $600 \mathrm{mg}$ of the IL-7R $\alpha$ blocking antibody or equivalent control injection. Pups were analyzed 9 days after final injection and cellularity of macrophages in the liver, lung, brain, and epidermis were determined.

B, Representative FACS plots and associated quantification of cellularity of tissue macrophages $\left(\mathrm{F} 4 / 80^{\mathrm{hi}} \mathrm{CD} 11 \mathrm{~b}^{\mathrm{lo}}\right)$ in the liver, lung, brain, and epidermis of neonates examined nine days following maternal injections of the A7R43 IL-7R $\alpha$ blocking antibody or IgG2A control administered at embryonic day (E)13.5 and E15.5. $\mathrm{N}=8-11$ representing three independent experiments. *, $\mathrm{P}<0.05$.

C, Representative FACS plots and associated quantification of cellularity of tissue macrophages $\left(\mathrm{F} 4 / 80^{\mathrm{hi}} \mathrm{CD} 11 \mathrm{~b}^{\text {lo }}\right)$ in the liver, lung, brain, and epidermis of IL7 $\mathrm{R}^{-/-}$and WT neonates at postnatal day (P) 19. $\mathrm{N}=4$ representing four independent experiments. *, $\mathrm{P}<0.05$.

D, A model for dynamic IL-7R $\alpha$ expression during fetal macrophage development. Fetal liver $\mathrm{F} 4 / 80^{\mathrm{lo}}$ monocytes express IL-7R $\alpha$ message, and mRNA expression is upregulated as $\mathrm{F} 4 / 80^{\mathrm{lo}}$ monocytes exit the liver and transit to peripheral tissues. However, upregulation of message is not associated with measurable surface expression in F4/80 ${ }^{\text {lo }}$ monocytes. IL $7 R \alpha$ surface expression only occurs upon differentiation of monocytes into $\mathrm{F} 4 / 80^{\text {hi }}$ macrophages in the tissues, at which point message levels dissipate. Continued message and surface expression postnatally within microglia and Langerhans cells account for higher levels of GFP labeling in those tissues, whereas GFP labeling is equilibrated by birth in the alveolar macrophages of the lung and Kupffer cells of the liver. 
Figure 1bioRxiv preprint doi: https://doi.org/10.1101/534859; this version posted January 30, 2019. The copyright holder for this preprint (which was not

A Flk2

IL-7Ra

Rag1
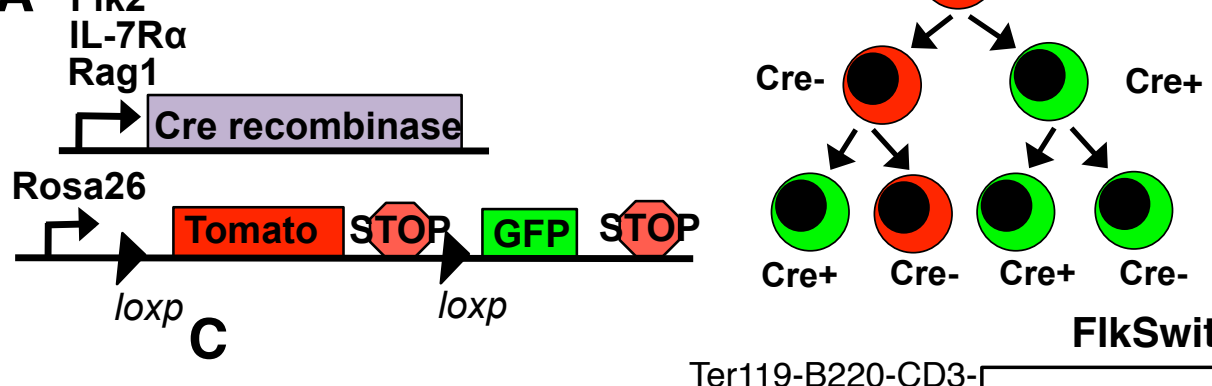

B

Cret Cre- Cre+ Cre-
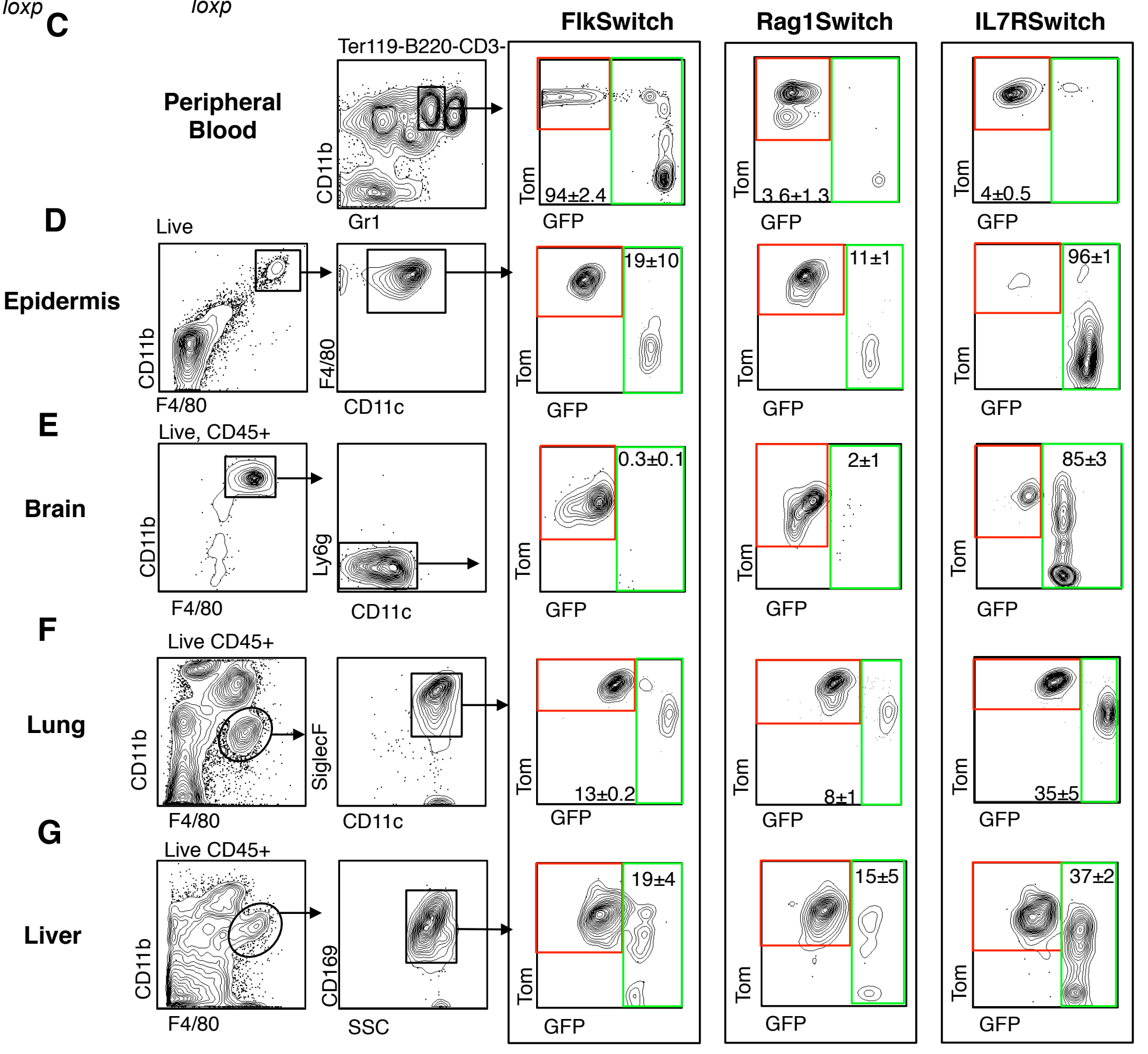

H
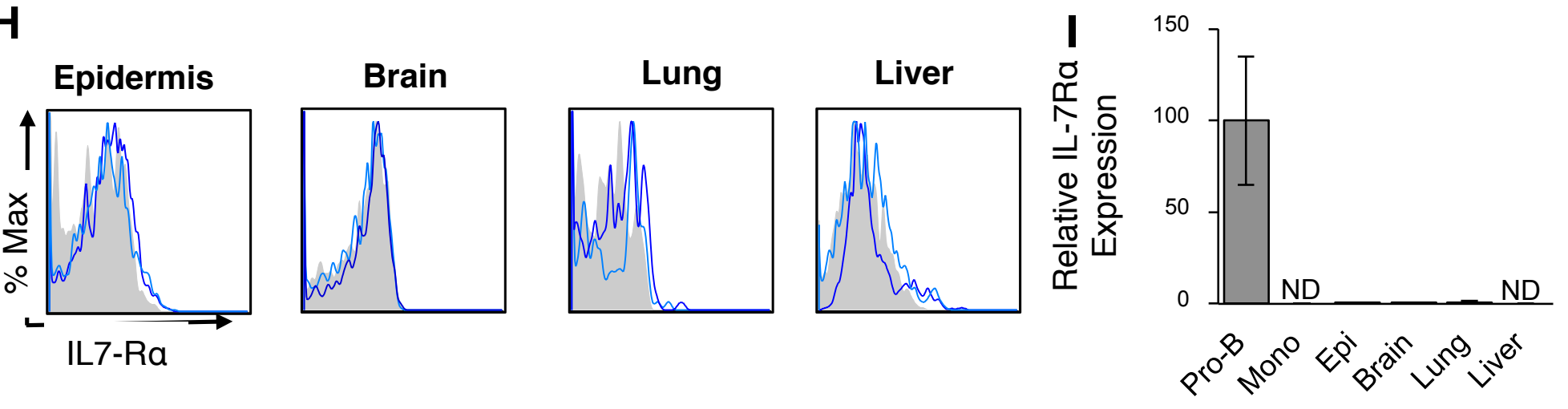
Figure 2pioRxiv preprint doi: https://doi.org/10.1101/534859; this version posted January 30, 2019. The copyright holder for this preprint (which was not certified by peer review) is the author/funder. All rights reserved. No reuse allowed without permission.

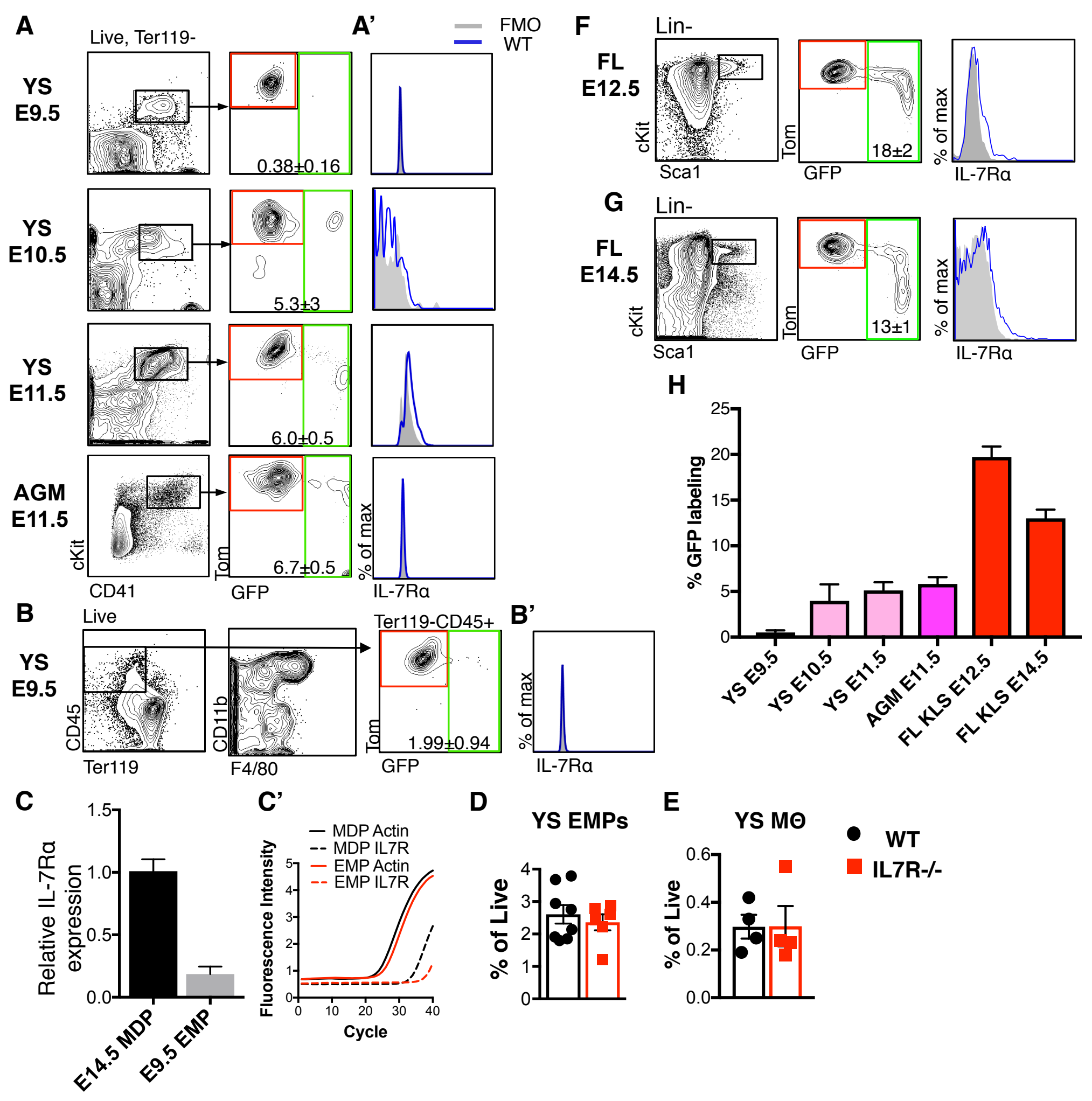



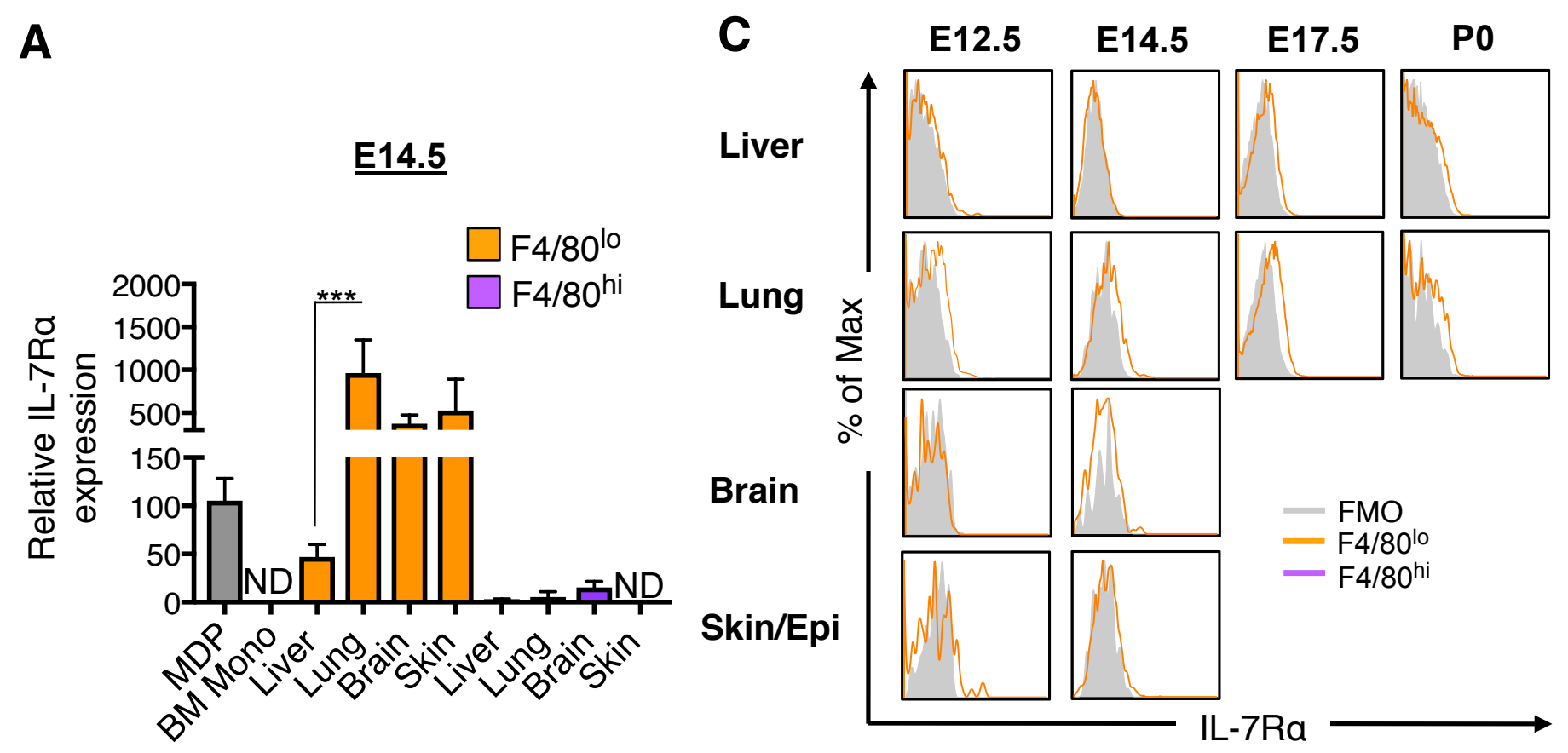

B

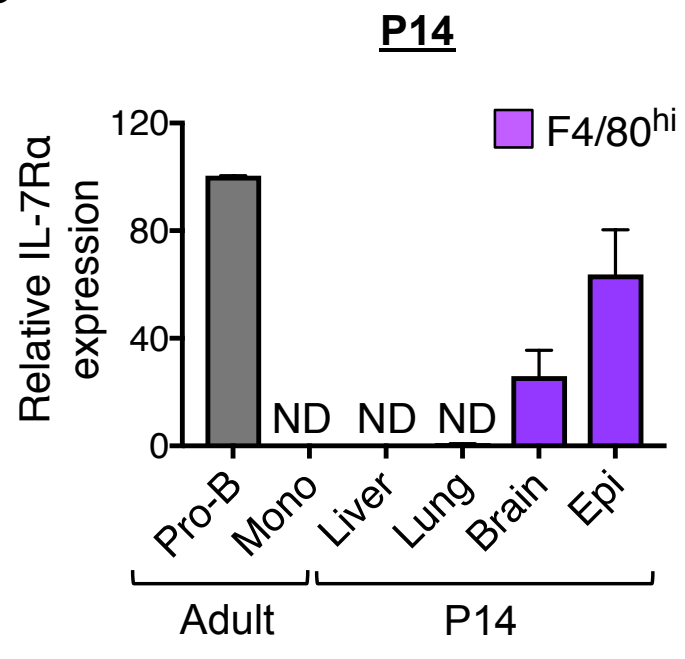

D

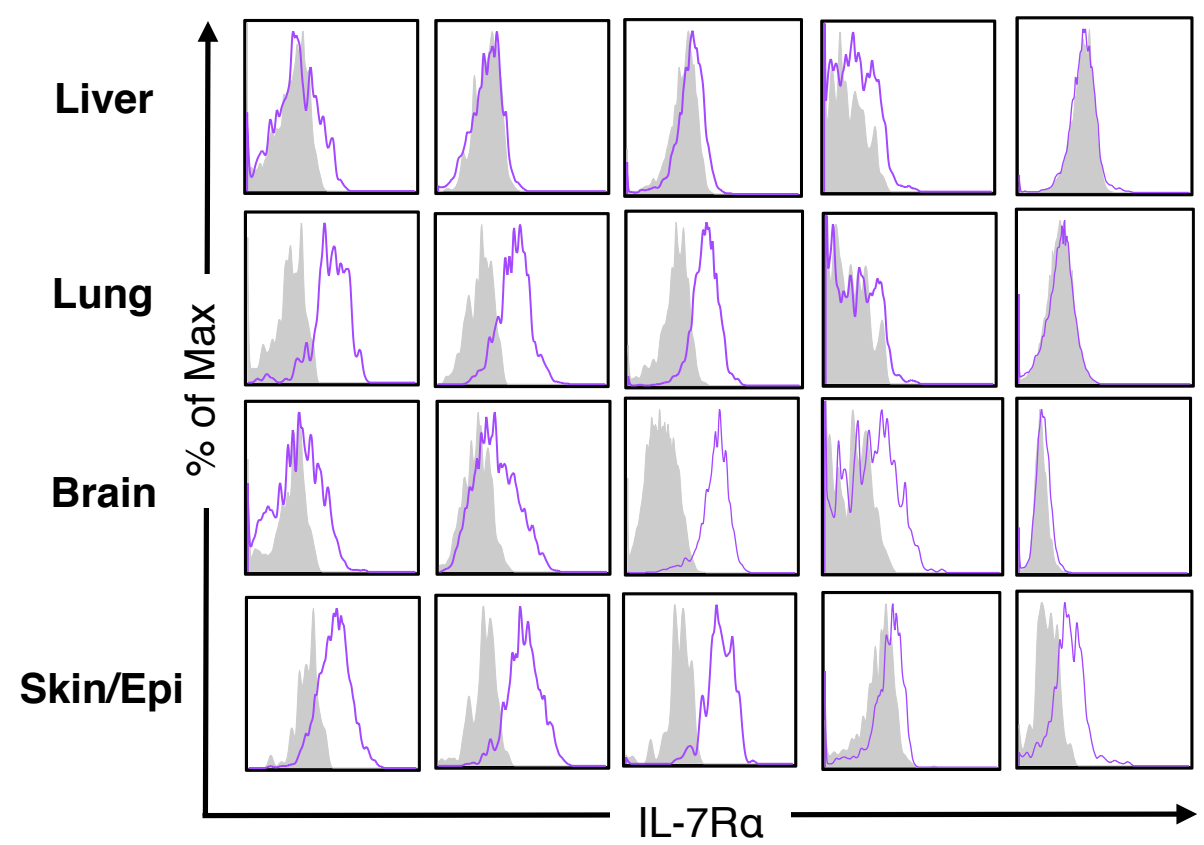


Figure Exiv preprint doi: https://doi.org/10.1101/534859; this version posted January 30, 2019. The copyright holder for this preprint (which was not certified by peer review) is the author/funder. All rights reserved. No reuse allowed without permission.

A Pre-plating

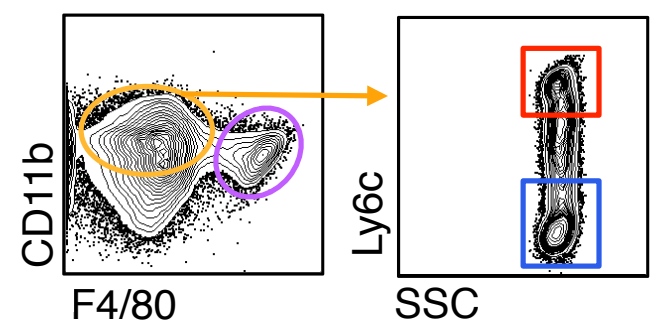

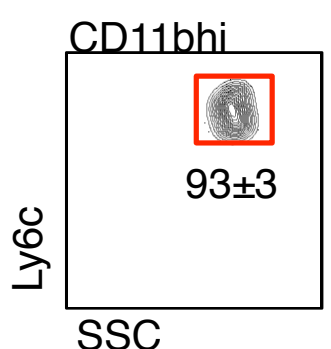

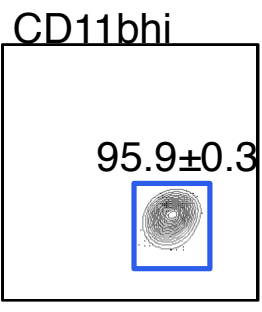

B
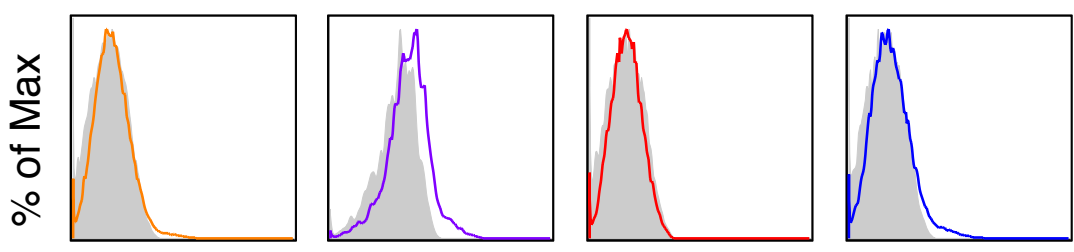

IL-7Ra

C

E

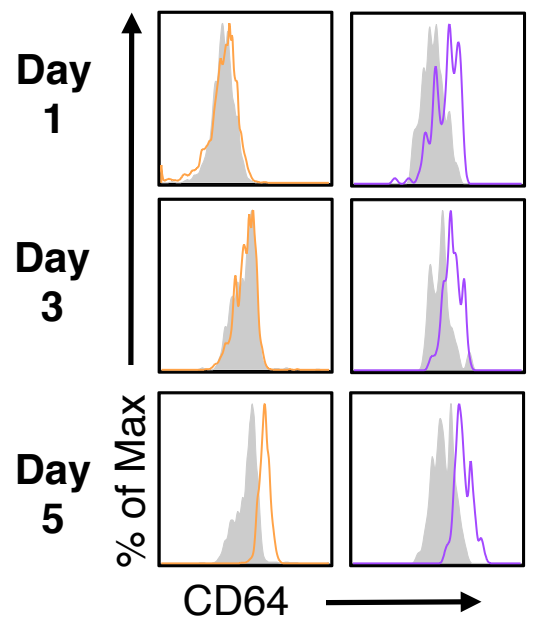

D'

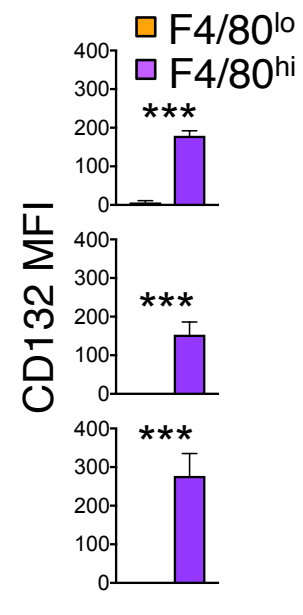

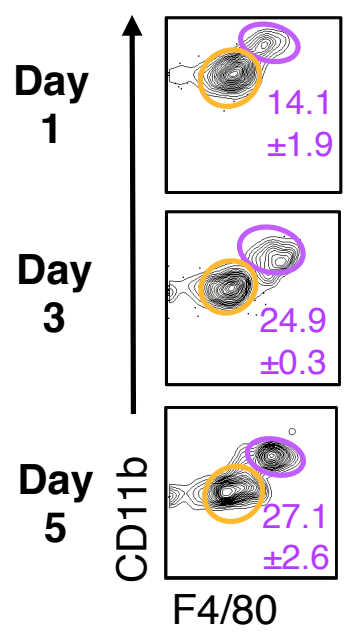

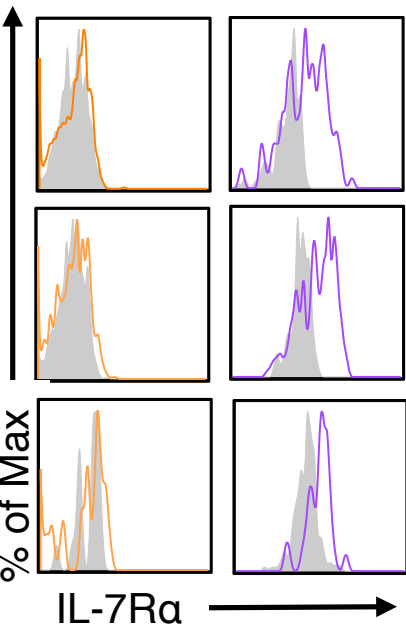

$E^{\prime}$
C

D

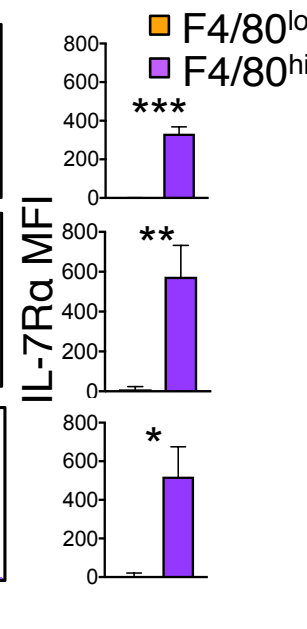

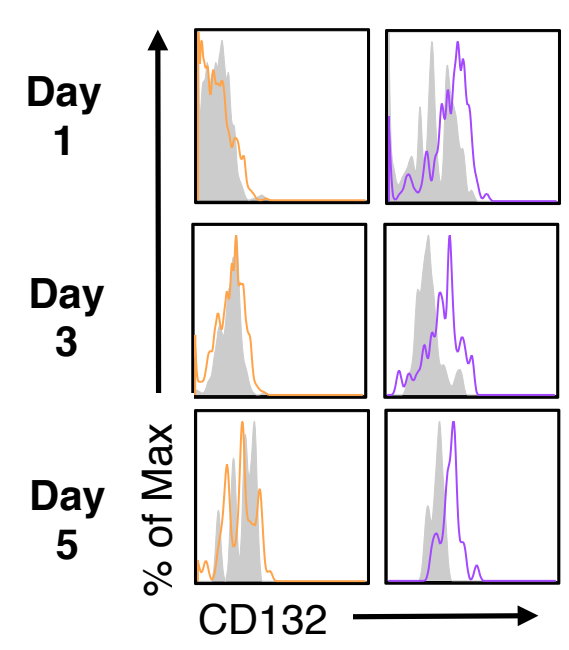

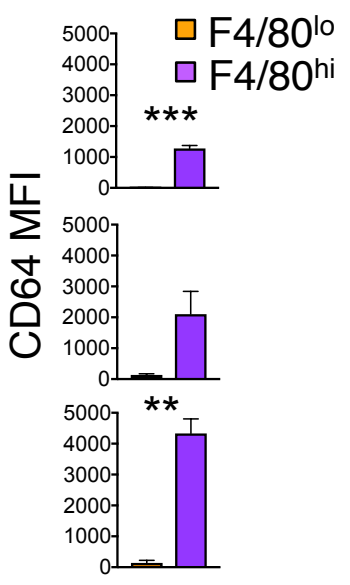


Figur@i@iviv preprint doi: https://doi.org/10.1101/534859; this version posted January 30, 2019. The copyright holder for this preprint (which was not certified by peer review) is the author/funder. All rights reserved. No reuse allowed without permission.

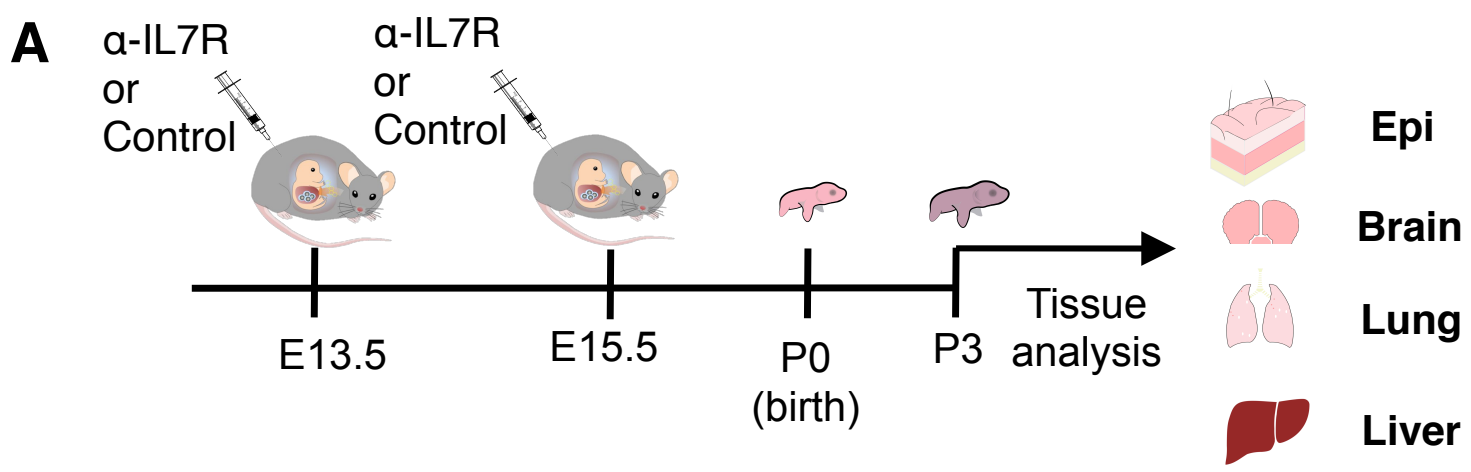

B

P3
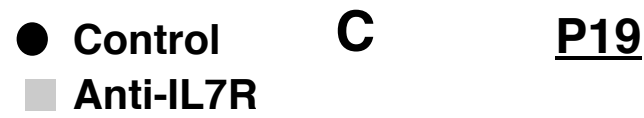

WT

Anti-IL7R

IL7R-/-
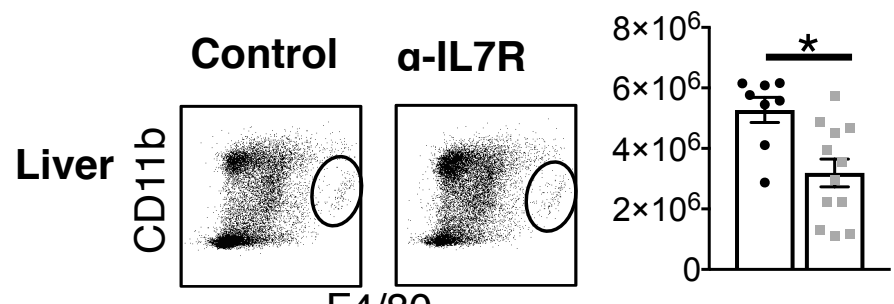

Lung $\frac{0}{\frac{1}{0}}$

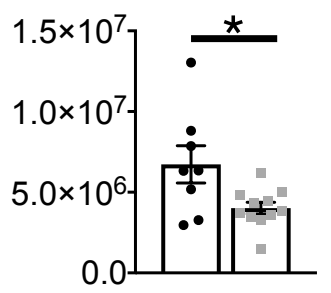

F4/80
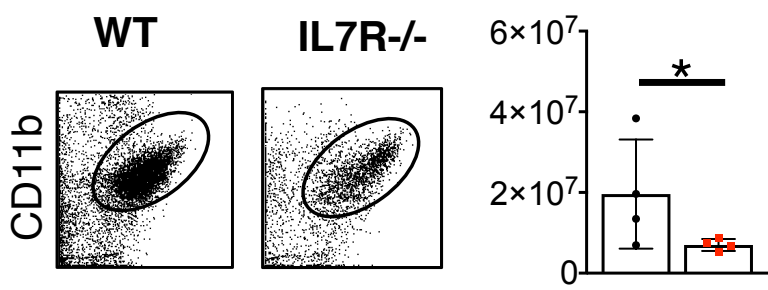

F4/80
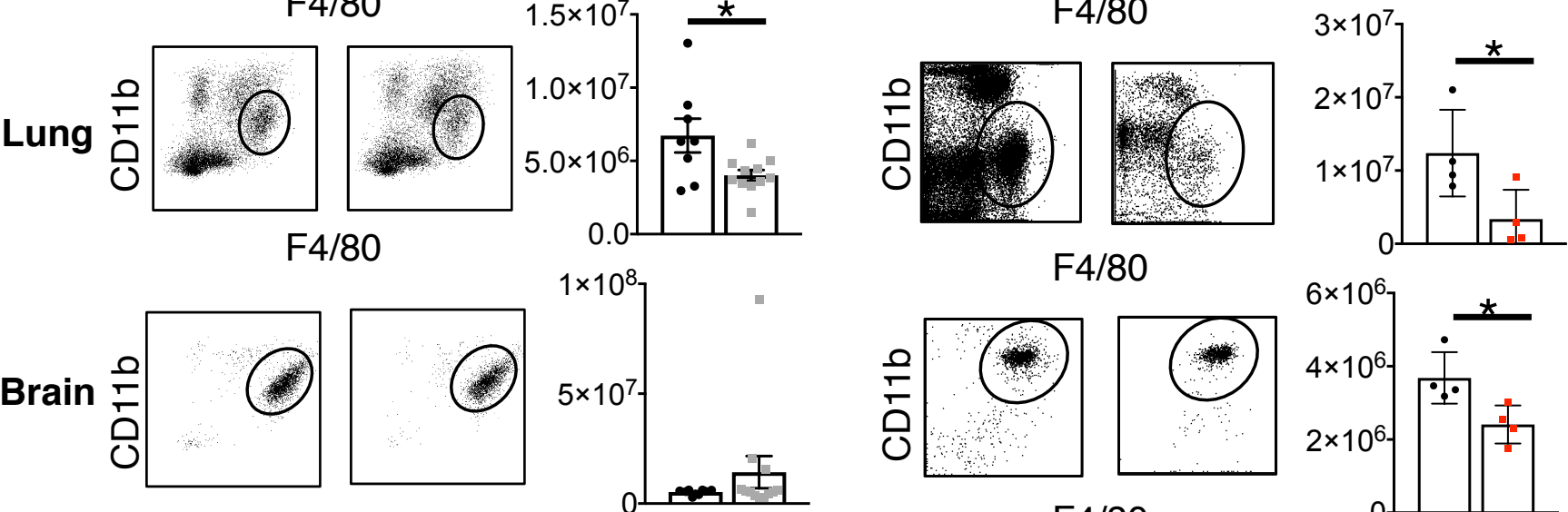

F4/80

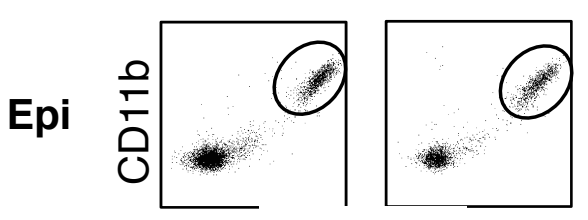

F4/80
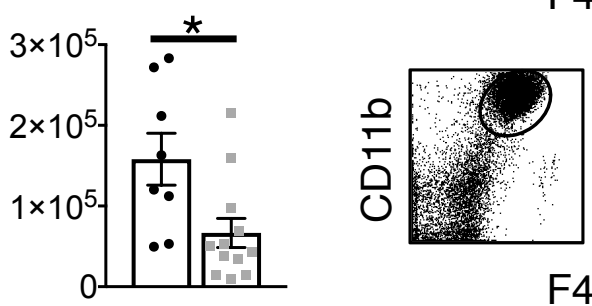

F4/80

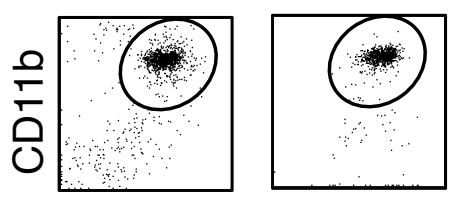

F4/80
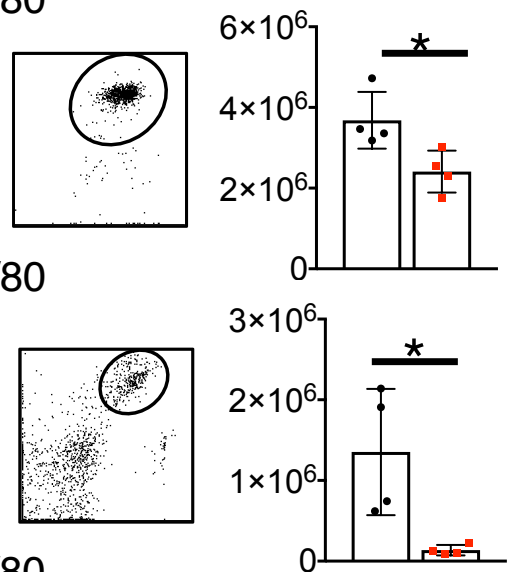

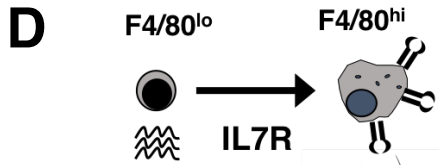

Epi

Brain 